bioRxiv preprint doi: https://doi.org/10.1101/2020.05.23.112250; this version posted May 25, 2020. The copyright holder for this preprint (which

was not certified by peer review) is the author/funder, who has granted bioRxiv a license to display the preprint in perpetuity. It is made available under aCC-BY-NC 4.0 International license.

\title{
1 Transcriptional re-wiring by mutation of the yeast Hsf1 oligomerization domain
}

2 Elizabeth A. Morton ${ }^{1 *}$, Michael W. Dorrity ${ }^{1 *}$, Wei Zhou ${ }^{1}$, Stanley Fields $^{1,2 \dagger}$, Christine Queitsch ${ }^{1 \dagger}$

$3 \quad{ }^{1}$ Department of Genome Sciences, University of Washington, Seattle, WA 98195

$4 \quad{ }^{2}$ Department of Medicine, University of Washington, Seattle, WA 98195

5

$6{ }^{*}$ These authors contributed equally to this work

$7 \dagger$ Correspondence to: Stanley Fields (ields@uw.edu) and Christine Queitsch (queitsch@uw.edu)

\section{8}

9

10

11

12

13

14

15

16

17

18

19 
20 Abstract: Response to heat stress is mediated by heat shock transcription factors (HSFs), which

21 possess conserved DNA-binding and oligomerization domains. The oligomerization domain is

22 required for HSF1 to transition under heat stress from a monomer to a homotrimer, which alters

23 DNA-binding specificity and affinity. Sequence variation in the oligomerization domain affects

24 HSF1 activity, although this link is poorly understood. We performed a deep mutational scan of

$25>400,000$ variants of the oligomerization domain of Saccharomyces cerevisiae Hsfl and

26 measured fitness under stress and non-stress conditions. We identify mutations that confer

27 temperature-specific phenotypes; some exceptional Hsflvariants lead to enhanced growth under

28 heat stress and changes to in vivo DNA-binding and transcriptional programs. The link between

29 Hsfl oligomerization and DNA-binding domain is evolutionarily conserved, with co-evolving

30 residues between these domains found among fungi. Mutation of transcription factor

31 oligomerization domains may represent a path toward re-wiring transcriptional programs without

32 mutation of DNA-binding domains. 


\section{Introduction}

36 The heat shock transcription factor (HSF1) is the central regulator of heat shock-inducible gene

37 expression and the cytoplasmic unfolded protein response in eukaryotes. HSF1 has two highly

38 conserved domains, mediating DNA-binding and oligomerization. ${ }^{1,2}$ In standard models of HSF 1

39 regulation, the protein is predominantly monomeric and inactive in the absence of stress. Upon

40 stress exposure, HSF1 becomes post-translationally modified and trimerizes into an active state

41 that upregulates heat shock target genes.

42 Despite half a century of study, the mechanisms by which HSF1 functions in the cell are not well

43 characterized beyond this simple model. HSF1 is essential in many model organisms, including

44 C. elegans, D. melanogaster, S. pombe and S. cerevisiae..$^{3-7}$ Beyond regulating heat shock, HSF1

45 contributes to development and fertility, ${ }^{3-5,8,9}$ aging, ${ }^{10}$ cancer, ${ }^{11-14}$ innate immunity, ${ }^{15,16}$ and

46 metabolism. ${ }^{17-20}$ Consistent with these multiple roles, the transcriptional programs mediated by

47 HSF1 differ in stress and non-stress conditions. ${ }^{12,21,22}$ The HSF1 DNA-binding domain is

48 remarkably conserved, showing few changes from yeast to humans. Though initial studies of $S$.

49 cerevisiae Hsf1 suggested it is constitutively trimerized and bound to DNA, ${ }^{7}$ more recent studies

50 indicate that Hsf1 substantially increases its target occupancy upon heat shock, similar to higher

51 eukaryotes in its temperature-dependent regulation of DNA binding. ${ }^{23,24}$

52 Beyond regulating homotrimerization, the HSF1 oligomerization domain mediates other inter-

53 and intramolecular interactions. ${ }^{25-31}$ Others have proposed that oligomerization of HSF1 can

54 affect specificity of DNA binding, ${ }^{28,32}$ and in yeast, oligomerization-defective phenotypes can be

55 suppressed by mutation in the DNA-binding domain. ${ }^{28}$ The oligomerization domain of HSF1 is

56 characterized by hydrophobic residues with heptad periodicity. Although this domain is 
57 commonly depicted as a single continuous domain, a region in the middle deviates from the ideal

58 hydrophobic heptad repeat, although it maintains the periodicity of the flanking N- and C-

59 terminal regions. Interruption of the hydrophobic pattern by this region, which we refer to as the

60 HR-spacer, initially suggested that the oligomerization domain contains two distinct $\alpha$-helices

61 (HR-A and HR-B). ${ }^{33,34}$ In vitro studies of Hsfl protein have shown that HR-A and HR-B show

62 differences in conformational change upon heat shock. ${ }^{26}$

63 We present here a deep mutational scan of a region of the oligomerization domain of $S$.

64 cerevisiae Hsfl that includes the C-terminus of HR-A, the HR-spacer, and most of HR-B. This

65 comprehensive sequence-function map of more than 400,000 variants allowed us to expand on

66 the role of this domain in DNA binding. Mutation of the oligomerization domain alone is

67 sufficient to alter both target binding and the downstream composition of its transcriptional

68 program. We identify a small subset of sites that can be mutated to confer exceptional stress

69 resistance, with the stress-resistant mutants showing a loss of temperature-dependent repression

70 of a subset of genes within the Hsf1 transcriptional program. These results suggest an

71 unexpected evolutionary path to tailor the activity of a transcription factor for different

72 environments by changing its oligomerization domain to regulate DNA binding rather than

73 changing the DNA-binding domain itself.

\section{Results}

75 Natural variation in the HR-A/B oligomerization domain affects fitness in a temperature-

76 specific fashion

77 In the consensus model of Hsfl regulation, Hsf1 trimerizes upon heat stress and binds to heat

78 shock elements (HSEs) to promote target gene expression (Fig. 1A). S. cerevisiae Hsf1 
79 (schematic in Fig. 1B) is essential at both basal $\left(30^{\circ} \mathrm{C}\right)$ and heat stress $\left(37^{\circ} \mathrm{C}\right)$ temperatures, with

80 increasing temperature accompanied by shifts in oligomeric state and changes in target

81 specificity. ${ }^{23,35}$ The oligomerization domain of the yeast protein is comprised of HR-A (heptads

82 1-5, residues 344-378); HR-spacer (heptads 6-7, residues 379-392); and HR-B (heptads 8-9,

83 residues 393-403) (Fig. 1E).

84 To better understand how sequence variation in the oligomerization domain contributes to Hsf1

85 function, we first sought to identify patterns of sequence conservation among natural

86 oligomerization domain variants, and to test the effects of natural oligomerization variants via

87 domain swap experiments. We analyzed conservation of residues according to their predicted

88 helix position in the triple-stranded coiled-coil trimer model. In the seven-residue periodicity

89 (conventionally labeled $a$ through $g$ ), the core $a$ and $d$ positions form the hydrophobic face that

90 mediates coiled-coil interactions of the trimer. ${ }^{33,36,37}$ Like others, ${ }^{33,38,39}$ we observed stronger

91 conservation of these core positions compared to other helix positions (Fig. 1C). As exceptions,

92 some species show variation even at core positions, and nearly all species show a break in

93 conserved hydrophobic amino acids at these sites within the HR-spacer (Fig. 1D, S1A). The lack

94 of hydrophobic residues in core positions of the HR-spacer and conservation of hydrophobics

95 outside of the core positions in HR-B suggest that these regions may encode functions beyond

96 forming the canonical coiled-coil required for survival under heat stress.

97 To test if variation in this domain alone could alter the temperature-specific function of Hsf1, we

98 replaced the $S$. cerevisiae oligomerization domain (residues 342-403) with the oligomerization

99 domains of six fungi predicted to vary in trimerization propensity (Fig. 1E, S1B). The chimeric

$100 \mathrm{Hsfl}$ proteins were tested at $30^{\circ} \mathrm{C}$ and $37^{\circ} \mathrm{C}$ for their ability to mediate $S$. cerevisiae growth in a

101 yeast background in which the endogenous HSF1 was regulated to allow repression by a 
102 tetracycline derivative, anhydrotetracyclin (ATc). Substitution of the S. cerevisiae

103 oligomerization domain with one from another species altered growth rate in a temperature-

104 specific manner. At basal temperature, all oligomerization domains rescued S. cerevisiae Hsf1

105 function to at least some extent (Fig. 1F). At high temperature, oligomerization domains from

106 three species that natively grow poorly at this temperature resulted in two (M. irregularis and $M$.

107 sympodialis $)^{40,41}$ that caused poor growth of $S$. cerevisiae, and another $(C \text {. vishniacii })^{42}$ that fully

108 rescued (Fig. 1F). The oligomerization domain of the thermophile T. appendiculata ${ }^{43}$ did not

109 confer enhanced growth of $S$. cerevisiae under temperature stress (Fig. 1F). These results suggest

110 that Hsfl temperature sensitivity does not depend solely on the oligomerization domain.

111 Phylogenetic distance of the substituted domains from the $S$. cerevisiae domain does not explain

112 differences in phenotype (Fig. 1E), suggesting that specific patterns of sequence variation in the

113 oligomerization domain rather than predicted trimerization propensity influence temperature-

114 dependent Hsf1 function.

\section{Deep mutational scanning of the Hsf1 HR-A/B domain}

117 Because variation in the Hsfl oligomerization domain influences temperature-dependent fitness,

118 we sought to analyze a region of this domain by deep mutational scanning, an approach to

119 comprehensively interrogate the functional consequences of amino acid substitutions at each

120 position of a protein. We targeted a 36 amino acid region of the oligomerization domain

121 (residues 365-400) spanning heptads 4 through 8, a region that includes 14 residues of HR-A, the

122 intervening 14 residues of the HR-spacer, and 8 residues of HR-B (Fig. 2C). 
123 A library of HSF 1 variants was created by doped oligo synthesis, with a $2.5 \%$ chance of mutant

124 base incorporation at any given position. The doped oligos were inserted into a low-copy

125 plasmid bearing the rest of yeast HSF 1, and the library was transformed into yeast. Given Hsf1

126 essentiality (Fig. 2A), we used the strain allowing ATc repression of HSF1 (Fig. 2B, see

127 Methods), paired with complementation by a plasmid-encoded HSF1 variant. Endogenous $H S F 1$

128 expression was permitted during transformation and expansion of the library to reduce selective

129 pressure before we carried out the mutational screen. Some selection occurred before

130 endogenous $H S F 1$ repression, attributable to putative dominant negative mutants, identified as

131 early drop-outs (Fig. S2). Samples of the plasmid library before and immediately after

132 transformation, as well as following overnight growth, were collected and included in

133 downstream sequencing.

134 We carried out competitive fitness selections at three temperatures $\left(30^{\circ} \mathrm{C}, 35^{\circ} \mathrm{C}\right.$ and $\left.37^{\circ} \mathrm{C}\right)$. The

135 library culture was grown for 4 hours at $30^{\circ} \mathrm{C}$ in the presence of ATc to repress endogenous Hsf1

136 (Fig. 2B), and then split into three replicate cultures grown at each of the three temperatures for

13714 hours $\left(30^{\circ} \mathrm{C}\right.$ and $\left.35^{\circ} \mathrm{C}\right)$ or 17 hours $\left(37^{\circ} \mathrm{C}\right)$ (Fig. $\left.2 \mathrm{~B}\right)$. Variant frequency was determined by

138 high-throughput sequencing of the plasmids isolated from these output cultures and compared to

139 the frequencies in the initial plasmid library, generating a fitness score. Variants that caused a

140 fitness defect over the growth interval decreased in frequency compared to wild type, while those

141 with a fitness benefit increased. Wild-type sequence made up $21.1 \%$ of the library. The yeast

142 library contained 465,531 variants with at least 5 input reads, including 620 of 720 possible

143 single amino acid changes (Fig. 2B). Correlation in variant scores was high between the three

144 replicates at each temperature (Table S1). As expected, variants with nonsense mutations led to

145 severe growth defects (Fig. 2C) and those with synonymous codons formed a distribution around 
146 zero (wild-type fitness) (Fig. S3). Mutations to proline were largely as detrimental as stop codons

147 at all temperatures (Fig. 2C), consistent with an $\alpha$-helical structure necessary for Hsf1 function at

148 both high and low temperatures. We confirmed these fitness results by individually testing eight

149 strains with variants that conferred a range of growth phenotypes. Growth rates of these strains

150 relative to a strain bearing wild-type Hsfl correlated with deep mutational scanning scores (Fig.

$1512 \mathrm{D})$.

152 Helical structure is a strong signal in the deep mutational scanning data

153 We compared the mutational scanning data to the predicted $\alpha$-helical structure of the

154 oligomerization domain (Fig. 3A and B). Substitutions at the $a$ and $d$ positions, particularly to

155 small or charged residues that cannot mediate hydrophobic interactions, decreased fitness (Fig.

$1563 \mathrm{~A}$ and $\mathrm{B}$ ) at both $30^{\circ} \mathrm{C}$ and $37^{\circ} \mathrm{C}$. This effect was not observed at the other positions (Fig. S4),

157 with the exception of position $c$, which displayed sensitivity at $30^{\circ} \mathrm{C}$ to small or charged residues

158 (Fig. 3B). The effect of helix position was also evident in double mutants: variants with

159 combined mutations at $a$ and $d$ positions performed worse than those at random pairs of helix

160 positions (Fig. S5A, S5B). We used double mutants to calculate pairwise epistasis scores

161 between positions within the oligomerization domain. Sites enriched for epistatic interactions

162 differed between the $30^{\circ} \mathrm{C}$ and $37^{\circ} \mathrm{C}$ conditions (Fig. S5C, S5D), supporting the idea of different

163 conformational constraints in Hsf1 upon heat stress. The observation that sites contributing to

164 trimeric structure are similarly sensitive to mutation at $30^{\circ} \mathrm{C}$ and $37^{\circ} \mathrm{C}$ suggests that the

165 difference in Hsfl regulation at these temperatures may be due to more subtle changes in the

166 degree of trimerization, or to a mechanism other than increased trimerization, such as altered

167 intramolecular or intermolecular interactions. 
168 We examined the effect of proline mutations specifically in the HR-spacer. Although all single

169 mutations to proline in this region were detrimental, consistent with a required $\alpha$-helix, prolines

170 at some positions were less deleterious in the presence of a second mutation (Fig. 3C). Most

171 functional proline-containing double mutants have at least one residue located in the HR-spacer,

172 heptad 7 (Fig. 3D). We validated the ability of a substitution to a canonical hydrophobic residue

173 in HR-A (N372I, position $a$ ) to rescue a proline substitution (A392P) in HR-spacer. Although

174 A392P resulted in a 40\% growth defect relative to wildtype, addition of N372I eliminated this

175 growth defect almost entirely (Fig. 3E). The observation that the effects of proline mutations can

176 be reversed more readily in the HR-spacer than elsewhere suggests that the requirement for

177 helical structure is relaxed in this region of the oligomerization domain, consistent with distinct

178 functions of this domain beyond coiled-coil formation.

180 Exceptional Hsf1 variants increase fitness under temperature stress

181 A small class of variants led to a growth benefit compared to wild type at $37^{\circ} \mathrm{C}$ (Fig. $4 \mathrm{~A}$ ). We

182 regenerated seven of these variants, including six double mutants (Fig. 4C), and assayed their

183 effect on growth rate. The individual variants conferred growth rates up to $20 \%$ higher than wild

184 type at $37^{\circ} \mathrm{C}$, but less so at $30^{\circ} \mathrm{C}$. One variant did not reproducibly confer the increased growth

185 rate (Fig. S6A). We selected two of the variants, A382C and the double mutant M380K, M381I,

186 for further characterization and found they also conferred enhanced growth under a different

187 proteotoxic stress, exposure to ethanol (Fig. S7). We engineered a subset of the variants into the

188 endogenous HSF1 locus but observed no significant growth rate increase (Fig. S6B), likely a 
189 result of the better growth of yeast expressing HSF1 alleles from the chromosome than from

190 plasmids in the tet-off background.

191 Several of the mutations in the six validated variants lie between residues 380-387 in heptads 6

192 and 7. For the top variants conferring enhanced growth (enrichment scores $>1.5$ sd higher than

193 mean at $37^{\circ} \mathrm{C}$ ), we tallied the helix positions at which mutations were present. Mutations at the

194 predicted helix interface (position $d$ ) were severely depleted (Fig. 4B). However, mutations at

195 positions $b$ or $c$, predicted to be facing outward from the helix, were over-represented (Fig. 4B).

196 Over the five heptad repeats that were mutagenized, residue 366 (in the fourth heptad) and

197 residues 380 and 381 (in the sixth heptad) contributed the strongest signal of any $b$ and $c$

198 positions (Fig. 4D, M381 marked with “+”).

199 Although the pattern of many of the hydrophobic changes associated with the stress-resistant

200 phenotype is consistent with a trimerized Hsfl, notable exceptions to the $a$ and $d$ periodicity

201 occurred in heptad 4 and in the HR-spacer and HR-B. The abundance and location of these

202 variants in the HR-spacer indicate that this region contributes to regulation of stress response by

203 Hsfl.

205 Loss of temperature-dependent regulation at a small set of transcriptional targets is

206 associated with stress resistance of exceptional Hsf1 variants

207 We hypothesized that some of the mutations that conferred stress-resistant phenotypes altered the

208 expression level, regulation or identity of Hsfl target genes. Thus, we performed RNA-

209 sequencing on two strains with variants that improved growth at $37^{\circ} \mathrm{C}(\mathrm{M} 380 \mathrm{~K}, \mathrm{M} 381 \mathrm{I}$ and 
210 N369Y,E373K) and two independent strains expressing wild-type HSF1. Samples were collected

211 soon after exposure to heat $\left(30^{\circ} \mathrm{C}\right.$ or $37^{\circ} \mathrm{C}$ for $15 \mathrm{~min}$ or 2 hours; see Methods $)$ or just before

212 temperature shift but after endogenous HSF1 repression ("+ATc").

213 Transcriptional profiles of the two improved-growth variants showed high similarity to each

214 other in genes differentially expressed compared to wild type at $37^{\circ} \mathrm{C}$ (Fig. S8), prompting us to

215 pool the variants for analysis. There were few differences in gene expression between variants

216 and wild type at the $15 \mathrm{~min}$ timepoint; however, significant differences were observed at the 2

217 hour timepoint at both temperatures (Fig. 5A, 5B). Unexpectedly, those genes with significant

218 upregulation at $37^{\circ} \mathrm{C}$ in the variants relative to wild type overlapped those with significant

219 downregulation at $30^{\circ} \mathrm{C}$ (Fig. 5B, 5E, Table S3). Thus, the primary pattern of differentially

220 expressed genes in the variants is the loss of temperature-specific regulation and maintenance of

221 an intermediate expression level of these genes relative to wild-type yeast (schematic drawing in

222 Fig. 5E inset). The exceptional Hsf1 variants showed neither constitutive nor over-expression of

223 genes induced in the canonical heat shock response, either of which could plausibly confer

224 increased stress tolerance (Fig. S9A, S9B). Instead, the variants failed to downregulate a set of

225 genes - largely involved in cell wall, metabolism, Golgi-associated vesicles and cytoplasmic

226 stress granules - in a temperature-dependent manner (Fig. 5F, Table S4). The most highly

227 differentially expressed gene between variants and wild type at $37^{\circ} \mathrm{C}, D E D 1$, encodes a protein

228 that is globally required for yeast mRNA translation initiation and localizes to the stress granule

229 (Fig. 5B). 
232 To understand how mutations of the Hsf1 oligomerization domain, especially in the HR-spacer,

233 result in altered transcriptional profiles, we compared the DNA-binding patterns of strains with

234 wild-type Hsfl or variants. The canonical Hsf1 binding motif, the heat shock element (HSE),

235 consists of three tandem inverted repeats of the sequence nGAAn. HSEs vary in the number and

236 spacing of these triplet repeats among HSF1-bound genes, ${ }^{12,23,28}$ making oligomerization state a

237 possible mechanism to differentiate among HSF1 targets.

238 To characterize variant Hsfl DNA binding, we employed the calling card method. ${ }^{44,45}$ In this

239 method, in vivo expression of a fusion between a transposase and a transcription factor tags sites

240 bound by the factor via transposon insertion events. Each event is molecularly barcoded so that

241 independent binding events can be quantified. To examine changes in the sites bound under

242 temperature stress, we applied the calling card method to wild-type Hsf1 as well as to the stress-

243 resistant variant M380K,M381I, which falls in the HR-spacer region. Analysis of sequences

244 flanking (150bp) transposition insertions by AME assessment identified two different types of

245 HSEs among significantly enriched motifs at $37^{\circ} \mathrm{C}$ (Table S5). These two different types of

246 HSEs (Type I, the canonical HSE, and Type II, also known as a step-type HSE ${ }^{46}$ ) exhibited

247 different enrichment among target binding site sequences of wild-type Hsf1 and M380K,M381I

248 (Fig. 5D and Table S5). De novo motif identification also found differences in significantly

249 enriched motifs between M380K,M381I and wild type (Table S6). We conclude that a subset of

250 the Hsf1 targeting profile remained intact in M380K,M381I, but some types of sites, particularly

251 those with type II HSEs, differed.

252 We also identified the nearest gene on either side of each insertion site. Of genes associated with

253 calling-card insertion sites, there are 131 targets in common between $37^{\circ} \mathrm{C}$ wild type and

254 M380K,M381I, enriched for GO biological processes of protein folding and response to heat and 
260 insertion site. We interpret these results as evidence that mutations of the Hsf1 oligomerization 261 domain can alter the binding specificity of Hsf1.

262 However, we did not observe significant overlap between the differentially expressed genes

263 identified by RNA-seq and the genes residing near the insertion sites. For M380K,M381I at

$26437^{\circ} \mathrm{C}$, the upregulated genes (205 genes) overlap with the calling card genes (343) by only 14

265 genes. For wild type at $37^{\circ} \mathrm{C}$, the upregulated genes (401) overlap with the calling card genes

266 (673) by only 43 genes, and the downregulated genes (861) overlap with the calling card genes

267 by 124 genes. Other studies have also observed discrepancy between transcription factor binding

268 sites and gene expression, ${ }^{47-49}$ attributable to such complexities of gene regulation as long-range

269 interactions, expression changes in genes that are not direct Hsf1 targets, and the necessary but

270 not sufficient nature of transcription factor binding.

272 Natural variation in the Hsf1 oligomerization domain is consistent with its role in altering 273 transcriptional targets

274 In light of our observation that yeast fitness can be modulated by mutations in the $H S F 1$

275 oligomerization domain, we examined natural variation in this domain in yeast species capable 276 of growing under temperature stress. Using growth data for 785 industrial yeast species, ${ }^{50,51}$ we 
277 identified nine thermophiles (capable of growth at $40^{\circ} \mathrm{C}$ or above) with available sequence data

278 (Table S9). Residue 381, in which mutation can enhance growth under stress, shows altered

279 amino acid preference among the nine thermophiles (Fig. 6A), with a 7-fold enrichment of

280 hydrophobic amino acids compared to background; the consensus residue among non-

281 thermophilic fungi was alanine $(p=0.005)$. As residue 381 lies within the HR-spacer (heptad 6,

282 position $c$ ), a hydrophobic residue would not be expected to contribute to trimerization (Fig. 1C).

283 To test if the HR-spacer showed expected patterns of intramolecular interactions, we examined

284 within-domain co-evolution between heptads of the oligomerization domain across 1229 species

285 of fungi. The trimeric coiled-coil structure predicts that heptads share co-evolving residues due

286 to the conserved function of its quaternary structure. Consistent with this expectation, we find

287 co-evolving sites within the same or adjacent heptad for all heptads except heptads 1 and 3 (Fig.

288 S10B); the assumed structure of the Hsf1 trimer as an extended coiled-coil does not predict

289 longer range contacts than these. Nevertheless, we find co-evolution of heptad 4 (HR-A) with

290 both heptads 8 and 9 (HR-B), which extend over the HR-spacer. Co-evolution between residues

291 distant in primary sequence can indicate physical or functional links.

292 Because transcription factors typically regulate target specificity via their DNA-binding

293 domains, we would predict co-evolution between the Hsf1 DNA-binding and trimerization

294 domains if both contribute to target specificity. Indeed, we found that mutations in the

295 oligomerization domain co-vary with mutations in the DNA-binding domain, consistent with our

296 experimental findings that mutating the oligomerization domain suffices to alter Hsf1 target

297 specificity (Fig. 6B, 6C, S10C). We further analyzed co-evolution at the level of individual

298 heptads in the oligomerization domain but failed to find specific relationships between the HR- 
299 spacer or HR-B regions and the DNA-binding domain, suggesting that interaction between these

300 domains maybe involve the entire oligomerization domain (Fig. 6C).

\section{Discussion}

303 By characterizing the effects of more than 400,000 variants of Hsf1 on yeast growth in basal and

304 stress conditions, we found that the oligomerization domain exhibits different mutational

305 sensitivities in basal and stress conditions, and that patterns of epistasis also vary between these

306 conditions. These results suggest that altered interactions among individual subunits of the Hsf1

307 trimer contribute to temperature-specific function (Fig. 6D). Both mutational sensitivity data and

308 patterns of epistasis point to fewer mutationally-sensitive helix positions at $37^{\circ} \mathrm{C}$, consistent with

309 a rigid, trimeric coiled-coil. In contrast, unexpected mutational sensitivity on the outer helix face

310 and a more complex pattern of epistatic interaction at $30^{\circ} \mathrm{C}$ suggests that the functional Hsfl

311 unit, while trimeric, may depend on additional residues in the outer helix face for complete

312 activity (Fig. 6D). Additionally, we identified exceptional variants in this domain that confer

313 enhanced growth at $37^{\circ} \mathrm{C}$, a phenotype accompanied by changes in transcriptional program and

314 DNA targeting (Fig. 6E). Many of these variants are double mutants, such as M380K,M381I, for

315 which the single composite mutations alone did not confer the $37^{\circ} \mathrm{C}$ benefit in the screen (Fig.

316 2C). We propose that the HR-spacer and HR-B have an activity in addition to mediating trimer

317 formation. The exceptional variants appeared to have lost temperature-dependent regulation -

318 either directly or through an indirect target - of a suite of genes involved in metabolic processes,

319 transport, cell wall and cytoplasmic stress granules, likely due to the link between DNA-binding

320 domain variation and oligomerization domain variation. In this case, the variants have resulted in 
321 cells that maintain an intermediate expression level that does not change under stress, suggesting

322 loss of temperature-dependent regulation underlies their stress-resistant phenotype. We predict

323 that these specific mutations would not be beneficial under other conditions, such as a fluctuating

324 environment. The prevalence of HSF1 gene duplications, especially in plant species, allows the

325 exploration of novel regulatory programs by introducing variation in other copies. Indeed,

326 variable length (in residue multiples of seven) of the HR-spacer has been used to distinguish

327 between classes of HSFs present in plants. ${ }^{52}$

328 It is possible that mutations at non-canonical positions in the HR-spacer or HR-B affect Hsf1

329 function through intra-molecular interaction. This model is also consistent with studies noting

330 that HR-B may act repressively in Hsf1 functional assays. ${ }^{34,53,54}$ It is also possible that the

331 variants that confer exceptional growth promote a different multimerization state. Hsfl occurs in

332 oligomeric states higher order than a trimer; Drosophila HSF has long been considered

333 hexameric in its active state, with hydrophobic residues in HR-B in a register one removed from

334 the main register possibly involved. ${ }^{38}$ These data also do not rule out altered interactions of other

335 regulators of Hsfl or disrupted post-translational modification.

336 While re-wiring the Hsfl-dependent stress-responsive regulatory network may be advantageous

337 under some conditions, maintaining the core heat-stress program is necessary for survival (Fig

338 6D). Similar rewiring of the Hsfl transcriptional program has been observed in cancer cells,

339 where Hsf1-dependent regulation and translation and metabolism genes drive cell growth in a

340 way that is distinct from heat stress-dependent regulation. ${ }^{12,55}$ There are parallels between the

341 effects seen in cancer cells and those we observe: changes to Hsf1 regulation have led to a "pro-

342 survival" phenotype that emphasizes cell growth even in the presence of a strong stress signal. 
343 We suggest that more subtle changes to the Hsfl regulatory network can be made through

344 mutations in the trimerization domain than through mutations in the DNA-binding domain itself.

345 Hsfl has flexibility in recognition of its DNA-binding site, with different spacings between

346 frequently degenerate GAA motifs. ${ }^{32,46,56}$ For this reason, the potential set of binding targets for

347 Hsfl is large compared to transcription factors with longer sequence motifs and less variability in

348 their arrangement. Furthermore, the requirement of a trimeric structure for high-affinity binding

349 to DNA imposes a constraint on mutations that alter affinity for DNA; the binding affinity

350 consequence of any single change to the DNA-binding domain is three-fold in the Hsf1 trimer.

351 Nevertheless, re-wiring of transcriptional networks is rampant in evolution, and altering the

352 transcriptional targets of Hsfl would be especially advantageous in changing how specific sets of

353 genes are regulated by temperature. Changes to the oligomerization domain may represent a

354 more viable path toward subtly altering transcriptional targets of Hsf1 (Fig. 6D) without overtly

355 compromising the underlying heat shock response. The possibility that new transcriptional

356 options are exposed by modification of the oligomerization domain has broader implications for

357 the many ways in which species can adapt transcription factor function over evolutionary time. ${ }^{57-}$

$358 \quad 61$

360 Methods

361 Deep mutational scan of S. cerevisiae HSF1 oligomerization domain.

362 The HSF 1 gene of S. cerevisiae was cloned under its native promoter (664bp upstream of the

363 start codon through 693bp downstream of the stop codon, see Table S10) into vector pRS415

364 (LEU2 selection). ${ }^{62}$ In order to facilitate large scale library cloning, silent restriction enzyme 
365 sites were introduced on either end of the target region - AvrII (CCTAGG) at base pairs 1041-

3661046 and Bpu10I (GCTTAGG) at base pairs 1244-1250 - to generate plasmid pEM74. The

367 region targeted for mutagenesis was base pairs 1093-1200 of the HSF1 coding sequence. A

368 doped oligo library of 168 bases was ordered (TriLink Biotechnologies) containing 30 bases of

369 invariant flanking sequence on either side of the 108 bases of the doped target region $(2.5 \%$ base

370 misincorporation). pEM74 was digested sequentially for 7.5hr each with AvrII and Bpu10I and

371 purified (Zymo Clean \& Concentrator D4003) to generate the vector component in which to

372 clone the doped oligo library. Flanking sequences in the doped oligo library were extended with

373 primers to increase overlap with the digested pEM74 (Table S10, the library was amplified with

374 primers EM244 and EM245 for 9 cycles $\left.98^{\circ} \mathrm{C} 15 \mathrm{sec}, 60^{\circ} \mathrm{C} 15 \mathrm{sec}, 70^{\circ} \mathrm{C} 15 \mathrm{sec}\right)$. Gibson assembly

375 (NEB \#E2611S) was employed to insert the amplified library into the AvrII- and Bpu10I-digsted

376 pEM74. The ligated variant library was cloned into DH10B cells (Invitrogen 18290015), grown

377 in bulk, purified, and transformed using lithium acetate (see below) into tet-off $H S F 1$ yeast

378 (KanMX::tetO::minpromoter::HSF1; URA3::CMV::tTA; his3-1;leu2-0;met15-0; MATa) ${ }^{63}$ Post-

379 transformation, the yeast library was recovered overnight in $400 \mathrm{~mL}$ C-Leu media, concentrated,

380 and frozen in $25 \%$ final concentration glycerol.

381 The yeast library was subjected to selection and screening conditions as follows. One hundred

382 milliliters of C-Leu media was inoculated with $\sim 1.2 \times 10^{8}$ cells of frozen yeast library stock and

383 grown at $30^{\circ} \mathrm{C}$ overnight. The culture was back-diluted to $100 \mathrm{~mL}$ of OD600 0.45 , at which point

384 the remaining overnight culture was pelleted and frozen as "overnight" timepoint. The back-

385 diluted culture was grown at $30^{\circ} \mathrm{C}$ for $2 \mathrm{hr}$, anhydrotetracycline (ATc) (Sigma-Aldrich 37919-

$386100 \mathrm{MG}-\mathrm{R}$ ) was added to $0.1 \mu \mathrm{g} / \mathrm{mL}$, and the culture was grown at $30^{\circ} \mathrm{C}$ for another $4 \mathrm{hr}$. The

387 culture was diluted to OD600 0.04 in three flasks of $100 \mathrm{~mL}$ C-Leu $+0.1 \mu \mathrm{g} / \mathrm{mL}$ ATc. A sample 
388 was taken as the "+ATc" timepoint. Flasks were put shaking at $200 \mathrm{rpm}$ at temperatures $30^{\circ} \mathrm{C}$,

$38935^{\circ} \mathrm{C}$, or $37^{\circ} \mathrm{C}$ and grown $14.5 \mathrm{hr}\left(37^{\circ} \mathrm{C}\right.$ was grown $\left.17.5 \mathrm{hr}\right)$. After growth, the $100 \mathrm{~mL}$ cultures

390 were spun down and pellets were frozen as post-selection timepoints.

391 Plasmids were extracted from pre- and post-selection libraries, as well as a sample from the

392 frozen library stock, using the Zymoprep Yeast Plasmid Miniprep II kit (D2004). These

393 minipreps were treated to remove genomic DNA contamination with combined Exonuclease I

394 (NEB M0293L) and Exonuclease III (NEB M0206L) for $30 \mathrm{~min}$ at $37^{\circ} \mathrm{C}$, followed by heat

395 inactivation at $95^{\circ} \mathrm{C}$ for 2 minutes.

396 Each plasmid miniprep sample was amplified by Phusion (NEB M0530L) to generate libraries of

397 the mutagenized HSF1 region flanked by cluster generating Illumina adaptors. Each sample was

398 amplified with a different index sequence in the primers for subsequent demultiplexing (Table

399 S10). Library amplified products were 320bp (including adaptor sequence). Following

400 amplification, libraries were Zymo Clean \& Concentrator purified (Zymo Research D4003),

401 eluting in 15L of nuclease-free water. Libraries were quantified with Qubit high sensitivity

402 (Invitrogen Q32854) and diluted to 2nM. Final library denaturation and dilution was performed

403 as described (NextSeq Denature and Dilute Libraries Guide 15048776 Rev. D). Libraries were

404 sequenced with a NextSeq High v2 300 Cycle kit (FC-404-2004), allowing full paired end

405 coverage of the $108 \mathrm{bp}$ mutagenized region.

406

407 Analysis of deep mutational scanning data. 
408 Using ENRICH software, read counts for each variant before (input) and after selection (output)

409 were used to determine fitness of $H S F 1$ variants. Briefly, counts for a particular variant in the

410 input and output libraries were normalized by their respective read depths to determine

411 frequency in each, and a ratio of the output and input frequencies determine a variant's

412 functional score. Finally, enrichment scores are normalized by the enrichment of wild-type Hsfl

413 in each selection. Intramolecular epistasis scores (as in S5C) were calculated for all double

414 mutants where constituent single mutants were tested; the value of epistasis is defined as the

415 deviation of double-mutant's fitness score from the multiplied scores of its constituent singles.

416 Negative epistasis scores indicate that a mutation's effect is exacerbated by the presence of a

417 partner mutation, while positive epistasis scores indicate that a mutation's effect is diminished by

418 the presence of a partner mutation. Analyses of mutational frequencies (as in Fig. 4D) that

419 include single, double and higher order mutants were completed by comparing two sets of

420 trimerization sequences: 1. A query set ranked by fitness scores (fitness $>1.5$ standard deviations

421 above mean $37^{\circ} \mathrm{C}$ fitness for Fig. 4D) 2. A background set with neutral fitness scores (within 0.5

422 standard deviations of the mean in Fig. 4D). Within each set, we calculate the frequency of each

423 amino acid at each site to generate a frequency matrix. Enriched amino acid changes in the query

424 set are identified by calculating the ratio of the query set's frequency matrix to the background

425 set's matrix.

427 Analysis of HSF1 HR-A/B oligomerization domain conservation in fungal sequences

428 Using fungal genomes deposited into NCBI and from the fungal sequencing project at Joint

429 Genome Institute, we created a BLAST database of translated coding sequences and queried with

430 the $S$. cerevisiae Hsf1 protein sequence. Full protein sequences from BLAST hits $(1,229)$ were 
431 aligned using MUSCLE ${ }^{64}$ and used to determine conservation at each site in the mutated

432 segment. Plots showing relative conservation of each amino acid per site were generated with

433 Weblogo. ${ }^{65}$ Co-evolving pairs of sites were identified using the MISTIC ${ }^{66}$ tool, wherein mutual

434 information is calculated between amino acid identities at all site pairs.

436 Cloning of HSF1 variants.

437 Point mutations identified from the DMS were introduced into pEM74 by the following method:

438 inverse PCR was performed on plasmid pEM74 with primers that amplified outside the region of

439 the desired nucleotide change. Gibson assembly (NEB \#E2611S) was then performed with the

440 inverse PCR and a 60-base oligo containing each desired mutation (Table S10 for inverse PCR

441 primers and oligos, along with the list of all mutants independently cloned outside of the library).

442 All clones were confirmed with Sanger sequencing. HSF1 variant clones were then transformed

443 into the tet-off HSF 1 strain ${ }^{63}$ by "one-step" transformation. "One-step" transformation: $1 \mathrm{~mL}$ of

444 saturated culture was pelleted, and $1 \mu \mathrm{L}$ of plasmid DNA (approximately 200-400ng) added to

445 the pellet, followed by $75 \mu \mathrm{L}$ of one-step buffer [0.1M lithium acetate, $38 \%$ PEG-3350, $0.1 \mathrm{M}$

446 DTT, $0.5 \mathrm{mg} / \mathrm{mL}$ carrier DNA (Roche 1467140)], after which the cells were vortexed, incubated

447 in a $42^{\circ} \mathrm{C}$ water bath for one hour, and plated on C-Leu selection plates. The $\Delta$ tri mutant was

448 created by ligation (using KLD enzyme mix NEB E0554S) of an inverse PCR that omitted the

449 HR-A/B domain (amino acids 342-403) (Table S10 for inverse PCR primers).

450 S. cerevisiae HR-A/B domain (amino acids 342-403) were replaced with HR-A/B domains of

451 other fungal species by inverse PCR and Gibson assembly with synthesized gBlocks of the

452 domains (Table S10). The HR-A/B amino acid coordinates of the species used: Cryptococcus 
453 vishniacii(510-571), Mucor irregularis (305-366), Malassezia sympodialis(142-203),

454 Microsporumgypseum (99-160), Thielaviaappendiculata (288-349), Chalara longipes (314-375).

456 Growth validation assays of HSF1 variants.

457 Variants identified in the DMS were validated in a plate reader growth assay. HSF 1 variants

458 expressed under the native $S$. cerevisiae promoter on a pRS415 backbone were transformed into

459 a background of tet-off $H S F 1$ (see above). Strains were cultured overnight at $30^{\circ} \mathrm{C}$ in C-Leu

460 media and backdiluted in the morning to an OD600 of 0.45. Backdiluted cultures were incubated

461 at $30^{\circ} \mathrm{C}$ for $2 \mathrm{hr}$, after which ATc was added to a final concentration of $0.1 \mu \mathrm{g} / \mathrm{mL}$. The cultures

462 were incubated for another $4 \mathrm{hr}$ to allow time for endogenous HSF1 repression. Strains were

463 backdiluted in C-Leu+0.1 $\mu \mathrm{g} / \mathrm{mL}$ ATc to an OD600 of 0.1 in a $200 \mu \mathrm{L}$ volume per well in 96 -well

464 culture plates. Eight technical replicate wells per strain per plate were run. Plates were sealed

465 with a plate cover and put in a BioTek Synergy $\mathrm{HI}$ microplate reader set to $30^{\circ} \mathrm{C}$ or $37^{\circ} \mathrm{C} .30^{\circ} \mathrm{C}$

466 and $37^{\circ} \mathrm{C}$ plates for each experiment were prepared together and run simultaneously on two

467 different microplate readers. Plates were incubated with orbital agitation for $\geq 23$ hr with 660

468 absorbance readings taken every 10min. Figure 4C represents eight technical replicates of one set

469 of transformants. An independent set of transformants is presented in supplemental Figure S6A.

470 The set of variants presented in Figure $2 \mathrm{C}$ was performed in tet-off $H S F 1$ yeast also containing

471 an SSA4 promoter driving GFP on a pRS413 backbone and so were cultured in C-Leu-His media

472 with ATc.

473 Exposure to ethanol stress was performed by growing above plasmid-expressed HSF1 variants in

474 a background of tet-off HSF1 overnight. Cultures were backdiluted to OD600 0.45, grown at 
$47530^{\circ} \mathrm{C}$ for $2 \mathrm{hr}$, after which ATc was added to a final concentration of $0.1 \mu \mathrm{g} / \mathrm{mL}$ and the cultures

476 were grown for another 4hr. Cultures were back-diluted again to OD600 0.1 in the presence of

$4770.1 \mu \mathrm{g} / \mathrm{mL}$ ATc and one of the following ethanol conditions: $0 \%, 5 \%, 8 \%$, or $10 \%$. As above,

478 these dilutions were grown in a BioTek Synergy HI microplate reader set to $30^{\circ} \mathrm{C}$ or $37^{\circ} \mathrm{C}$. Four

479 technical replicate wells were run for each variant and empty vector, and eight technical replicate

480 wells were run for the wild type Hsfl plasmid control.

483 HSF 1 sequence with point mutations were introduced into helper plasmid of PiggyBac-based

484 transposon system. ${ }^{45}$ Each helper plasmid was paired with a donor plasmid containing specific

485 barcode within transposon. All constructs were confirmed with Sanger Sequencing. Paired donor

486 plasmid and helper plasmid were transformed into yeast (BY4705 MAT $\alpha$ ) by high-efficiency

487 lithium acetate transformation. ${ }^{67}$ After transformation, cells were collected and put on the

488 induction plates with galactose. Cells were induced for 5 days in either $30^{\circ} \mathrm{C}$ or $37^{\circ} \mathrm{C}$ (heat shock

489 condition) to express the HSF1-PBase. Cells were then collected, diluted back to OD600=0.45,

490 and cultured in rich medium for 6 hours recovery (to OD600= 1.6). Cells were put onto

491 selection plates with 5-FOA and G418 at varying dilutions and grown 2-3 days. Cells were then

492 collected from selection plates and genomic DNA was extracted from each sample using the

493 Smash and Grab method as described. ${ }^{68}$ Four variants of HSF1 in addition to wild-type HSF1

494 were expressed for this assay and exposed to $30^{\circ} \mathrm{C}$ or $37^{\circ} \mathrm{C}$ in two replicates; all of the $H S F 1$

495 variant-expressing yeast failed to produce enough colonies for collection at $30^{\circ} \mathrm{C}$, therefore only

496 wild type had data for both $30^{\circ} \mathrm{C}$ and $37^{\circ} \mathrm{C}$. Other variants and wild-type had two replicates per

497 temperature, except $\mathrm{A} 382 \mathrm{P} 37^{\circ} \mathrm{C}$, where a single sample was collected. The library for each 
498 group were prepared through the protocol described by the calling card-seq method. ${ }^{45}$ The PCR

499 products were pooled and Illumina sequenced.

500 The raw data included all FASTQ files from the PiggyBac transposase-only control, wild type

501 HSF1 and the four variants: M380K,M381I (phenotype of enhanced growth at $37^{\circ} \mathrm{C}$ ),

502 M380I,M381K (mildly enhanced or approximately equal to wild type, Fig. S6A), Q389K (mildly

503 impaired growth), and A382P (severely impaired growth), two replicates each. In read1, the first

504 segment is the universal primer sequence: CGTCAATTTTACGCAGACTATCTTTCTAGGG

505 followed by the flanking genome sequence of $39 \mathrm{bp}$. The first $8 \mathrm{bp}$ of read 2 is the UMI sequence

506 used to identify unique insertion events. We first filtered sequence reads with high quality and

507 mapped them back to the yeast genome. Then we quantified independent PiggyBac insertions

508 based on the UMIs detected in each sample. Target genes were then assigned to insertion peaks

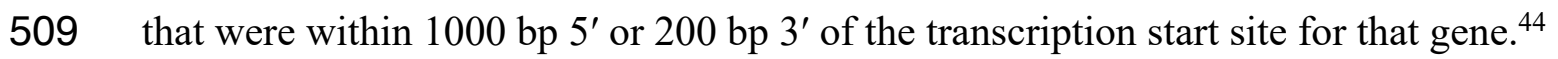

510 Insertions with counts above the 85 th percentile were identified as "high count insertions." We

511 identified $300 \mathrm{bp}$ windows around each high count insertion, and then merged the windows

512 (bedops $-\mathrm{m}$ ) to generate high insertion count sites for each replicate. We extracted the sequence

513 from these windows and attempted to identify de novo motifs using MEME (Table S6). ${ }^{69}$ We

514 used $\mathrm{AME}^{69}$ to determine enrichment of known motifs in the target sequences. For AME,

515 sequences (300bp: 150bp flanking each high-cut-count insertion site) were compared to a

516 reference database of motifs. ${ }^{70}$ Significance values are given as reported by AME; in summary,

517 for motif enrichment in target sequences p-values were calculated by one-tailed Fisher exact test

518 compared to occurrence in 2-mer shuffled control sequences, with Bonferroni correction for the

519 number of sequences input for each sample. E-values reported by AME represent p-values 
520 corrected for the expected number of motifs enriched in the test sequences given the testing of

521 multiple motifs. (AME results Table S5).

522 A gene target list was generated by identifying the closest gene on either side of the high-cu-

523 count insertion site. Both genes were included in the final gene list per sample, with duplicate

524 gene IDs removed (Table S7). The overlap in gene lists for the two replicates of a given

525 genotype was used as that genotype's final gene list. Gene Ontology enrichment was performed

526 using gprofiler (default parameters of g:SCS significance threshold multiple testing correction) ${ }^{71}$

527 RNA-seq of HSF1 variants.

528 RNA-seq was performed on yeast bearing $H S F 1$ variants transformed into tet-off $H S F 1$ yeast. ${ }^{63}$

529 Early timepoint RNA cultures were collected for transformants of plasmids bearing

$530 H S F 1(\mathrm{~N} 369 Y$, E373K), HSF1(M380K, M381I), or two independent clones of plasmid rescue

531 with wild-type HSF1 (all clones containing silent restriction sites). Clones were grown overnight

532 at $30^{\circ} \mathrm{C}$ in C-Leu media and back-diluted the following morning to OD600 0.45. These back-

533 dilutions were grown for $2 \mathrm{hr}$ at $30^{\circ} \mathrm{C}$ and then ATc was added to a final concentration of 0.1

$534 \mu \mathrm{g} / \mathrm{mL}$ and the cultures were given $4 \mathrm{hr}$ more at $30^{\circ} \mathrm{C} .25 \mathrm{~mL}$ of this culture was collected for the

535 "+ATc" sample. Cultures were diluted to OD600 $0.1 \mathrm{in} \mathrm{C-Leu}+0.1 \mu \mathrm{g} / \mathrm{mL}$ ATc in two sets of

536 flasks, one of which was placed at $30^{\circ} \mathrm{C}$ and the other at $37^{\circ} \mathrm{C}$. Samples of both $30^{\circ} \mathrm{C}$ and $37^{\circ} \mathrm{C}$

537 cultures were collected after $15 \mathrm{~min}$ and after $2 \mathrm{hr}$ at their respective temperatures. Collection of

538 each timepoint was done as follows: $50 \mathrm{~mL}$ of the cultures was spun $4000 \mathrm{rcf} 5 \mathrm{~min}$, the pellet

539 resuspended in the residual media and transferred to a $1.5 \mathrm{~mL}$ tube, then spun again at $21130 \mathrm{rcf}$

540 for $5 \mathrm{~min}$ and the supernatant removed, immediately after which the pellet was flash frozen in

541 liquid nitrogen. 
542 RNA was isolated via acid-phenol extraction. ${ }^{72}$ RNA was DNase treated with RQ1 DNase

543 (Promega M6101) by addition of $5 \mu \mathrm{L}$ RQ1 buffer and $5 \mu \mathrm{L}$ RQ1 DNase to each RNA sample,

544 incubation at $37^{\circ} \mathrm{C}$ for $45 \mathrm{~min}$ and then stopping the reaction with $5 \mu \mathrm{L}$ provided stop solution.

545 cDNA was generated with Superscript IV reverse transcriptase (Invitrogen 18090050). 5 $\mu \mathrm{g}$ of

546 RNA was combined with oligo $\mathrm{d}(\mathrm{T})_{18}$ primers and dNTPs according to manufacturer instructions

547 and incubated $65^{\circ} \mathrm{C} 5 \mathrm{~min}$. Reactions were put on ice $2 \mathrm{~min}$ and then combined with $4 \mathrm{~L} 5 \mathrm{X}$ SSIV

548 buffer, $1 \mu \mathrm{L} 100 \mathrm{mM}$ DTT, $1 \mu \mathrm{L}$ RiboLock RNase inhibitor (Thermo Fisher K1622), and $1 \mu \mathrm{L}$

549 SuperScript IV reverse transcriptase. The reaction incubated at $55^{\circ} \mathrm{C}$ for $15 \mathrm{~min}$ followed by

$55080^{\circ} \mathrm{C}$ for $10 \mathrm{~min}$ before being put on ice. Second strand synthesis was performed with the

551 NEBNext Second Strand Synthesis module (NEB E6111S). 48 $\mu$ L of nuclease-free water was

552 added to each tube, followed by $8 \mu \mathrm{L}$ of $10 \mathrm{X}$ Second Strand Synthesis reaction buffer and $4 \mu \mathrm{L}$

553 Second Strand Synthesis enzyme mix. The reaction incubated at $16^{\circ} \mathrm{C}$ for $2.5 \mathrm{hr}$, after which the

554 cDNA was purified using the Zymo Clean \& Concentrator kit (Zymo Research D4003).

555 Libraries were generated via tagmentation of cDNA (Illumina FC-121-1030) and sequenced.

556 Gene Ontology enrichment was performed using g:Profiler (default parameters of g:SCS

557 significance threshold multiple testing correction) and category list for Fig. 5F condensed with

558 REVIGO. ${ }^{71,73}$

561 Integration of $H S F 1$ variants at the endogenous locus was done using Gibson assembly (NEB

562 \#E2611S) to create clones of three HSF1 variants: A382C; M380K,M381I; and N369Y,E373K. 
563 These versions of HSF1 had otherwise wild-type sequence and were lacking the restriction sites

564 introduced in the cloning process of the original deep mutational screen (see above). 664bp of

565 promoter was included upstream of HSF1 along with $430 \mathrm{bp}$ of downstream sequence before a

$566 U R A 3$ cassette flanked by homologous recombination arms. ${ }^{74}$ The $H S F 1$ variant plus URA3

567 cassette was amplified using primers with an additional $43 \mathrm{bp}$ of $H S F 1$ downstream sequence

568 added to the 3' end (Table S10). This PCR product was transformed with lithium acetate into

569 yeast strain BY4741 and plated on C-Ura. Resulting recombinants were genotyped using PCR

570 and Sanger sequencing (Table S10). Strains with URA3 integration but wild-type HSF1 sequence

571 were also isolated as matched controls (Fig. S6B). Genotyped strains were then grown on 5-

572 fluoroorotoic acid (5-FOA, $1 \mathrm{~g} / \mathrm{L})$ to counter select for $U R A 3$ and generate a line with the uracil

573 auxotrophy. Genomic DNA was extracted (Zymo \#D2002) from colonies grown on 5-FOA and

574 Sanger sequenced to confirm presence or absence of HSF1 mutation and URA3 gene.

576 Lithium acetate transformation protocol

577 One hundred milliliters of culture was grown to approximately OD 1.8. The culture was pelleted

$5783 \mathrm{~min} 3000 \mathrm{rpm}$. Supernatant was removed and cells were resuspended in 15mL LiSorb (lithium

579 acetate, $1 \mathrm{M}$ sorbitol, Tris, EDTA). Cells were pelleted and resuspended in $15 \mathrm{~mL}$ LiSorb again,

580 and then pelleted and resuspended in $1.5 \mathrm{~mL}$ LiSorb. The cells were split between two $1.5 \mathrm{~mL}$

581 tubes and allowed to incubate with rotation at room temperature for $30 \mathrm{~min} .200 \mu \mathrm{L}$ of the cell

582 suspension was added to four tubes, each containing $50 \mu \mathrm{L}$ of $2 \mathrm{mg} / \mathrm{mL}$ salmon sperm DNA and

$5833 \mu \mathrm{L}$ DNA for transformation $(500 \mathrm{ng} / \mu \mathrm{L}$ DMS library or PCR product for integration(84 to

$584231 \mathrm{ng} / \mu \mathrm{L})$ ). $1 \mathrm{~mL}$ of LiPEG (lithium acetate, Tris, EDTA, 44\%PEG) was added and the tubes 
585 were allowed to incubate with rotation at room temperature for $30 \mathrm{~min}$. $100 \mu \mathrm{L}$ DMSO was added

586 and the tubes were incubated $42^{\circ} \mathrm{C}$ for $10 \mathrm{~min}$, after which the cells were pelleted $1 \mathrm{~min} 5000 \mathrm{rpm}$,

587 the supernatant removed, and the pellet resuspended in YPD+0.5M sorbitol. The culture was

588 allowed to recover at $30 \mathrm{C}$ for $1 \mathrm{hr}$. For PCR product transformation, the culture was then pelleted

589 and plated on C-Ura plates. For DMS library transformation, the cells were added to 400mL C-

590 Leu media and allowed to grow overnight before concentrating and freezing.

592 Data Availability

593 Expression data are available at the Gene Expression Omnibus (GEO number: pending).

\section{Acknowledgements}

596 We thank Dr. Kerry Bubb for valuable assistance in data processing. This work was supported

597 by the National Institutes of Health [Genetic Approaches to Aging Training Grant 4 T32 AG 57-

598 39] and the National Human Genome Research Institute [Genome Sciences Training Grant 5

599 T32 HG 35-19] to E.A.M., and also the NSF GRFP and WRF-Hall Fellowship to M.W.D. The

600 work was also supported by NIH grants GM114166 and 1RM1HG010461 to C.Q. and S.F.

602 References

603 1. Peteranderl, R. et al. Biochemical and biophysical characterization of the trimerization 
604 domain from the heat shock transcription factor. Biochemistry 38, 3559-69 (1999).

605 2. Sakurai, H. \& Enoki, Y. Novel aspects of heat shock factors: DNA recognition, chromatin 606 modulation and gene expression. FEBS J. 277, 4140-4149 (2010).

607 3. Hajdu-Cronin, Y. M., Chen, W. J. \& Sternberg, P. W. The L-type cyclin CYL-1 and the 608 heat-shock-factor HSF-1 are required for heat-shock-induced protein expression in 609 Caenorhabditis elegans. Genetics 168, 1937-1949 (2004).

610 4. Morton, E. A. \& Lamitina, T. Caenorhabditis elegans HSF-1 is an essential nuclear 611 protein that forms stress granule-like structures following heat shock. Aging Cell 12, 112$612120(2013)$.

613 5. Jedlicka, P., Mortin, M. A. \& Wu, C. Multiple functions of Drosophila heat shock 614 transcription factor in vivo. EMBO J. 16, 2452-2462 (1997).

615 6. Gallo, G. J., Prentice, H. \& Kingston, R. E. Heat shock factor is required for growth at 616 normal temperatures in the fission yeast Schizosaccharomyces pombe. Mol. Cell. Biol. 13, 617 749-761 (1993).

618 7. Sorger, P. K. \& Pelham, H. R. B. Yeast Heat Shock Factor Is an Essential DNA-Binding 619 Protein That Exhibits Temperature Dependent Phosphorylation. Cell 54, 855-864 (1988).

620 8. Xiao, X. Z. et al. HSF1 is required for extra-embryonic development, postnatal growth 621 and protection during inflammatory responses in mice. EMBO J. 18, 5943-5952 (1999).

622 9. Metchat, A. et al. Mammalian heat shock factor 1 is essential for oocyte meiosis and 623 directly regulates Hsp90 $\alpha$ expression. J. Biol. Chem. 284, 9521-9528 (2009). 
624 10. Hsu, A. L., Murphy, C. T. \& Kenyon, C. Regulation of aging and age-related disease by

625 DAF-16 and heat-shock factor. Science (80-. ). 300, 1142-1145 (2003).

626 11. Dai, C., Whitesell, L., Rogers, A. B. \& Lindquist, S. Heat Shock Factor 1 Is a Powerful 627 Multifaceted Modifier of Carcinogenesis. Cell 130, 1005-1018 (2007).

628 12. Mendillo, M. L. et al. HSF1 drives a transcriptional program distinct from heat shock to 629 support highly malignant human cancers. Cell 150, 549-562 (2012).

630 13. Scherz-Shouval, R. et al. The reprogramming of tumor stroma by HSF1 is a potent $631 \quad$ enabler of malignancy. Cell 158, 564-578 (2014).

632 14. Yin, J. et al. piR-823 contributes to colorectal tumorigenesis by enhancing the 633 transcriptional activity of HSF1. Cancer Sci. 108, 1746-1756 (2017).

634 15. Singh, V. \& Aballay, A. Heat-shock transcription factor (HSF)-1 pathway required for 635 Caenorhabditis elegans immunity. Proc. Natl. Acad. Sci. U. S. A. 103, 13092-7 (2006).

636 16. Inouye, S. et al. Impaired IgG production in mice deficient for heat shock transcription 637 factor 1. J. Biol. Chem. 279, 38701-38709 (2004).

638 17. Ma, X. et al. Celastrol protects against obesity and metabolic dysfunction through 639 activation of a HSF1-PGC1 $\alpha$ transcriptional axis. Cell Metab. 22, 695-708 (2015).

640 18. Minsky, N. \& Roeder, R. G. Direct link between metabolic regulation and the heat-shock 641 response through the transcriptional regulator PGC-1 2. Proc. Natl. Acad. Sci. U. S. A. 112, $642 \quad$ E5669-E5678 (2015).

643 19. Jin, X., Qiao, A., Moskophidis, D. \& Mivechi, N. F. Modulation of Heat Shock Factor 1 
Activity through Silencing of Ser303/Ser307 Phosphorylation Supports a Metabolic Program Leading to Age-Related Obesity and Insulin Resistance. Mol. Cell. Biol. 38, (2018).

647 20. Qiao, A., Jin, X., Pang, J., Moskophidis, D. \& Mivechi, N. F. The transcriptional regulator 648 of the chaperone response HSF1 controls hepatic bioenergetics and protein homeostasis. $J$. $649 \quad$ Cell Biol. 216, 723-741 (2017).

650 21. Li, J., Labbadia, J. \& Morimoto, R. I. Rethinking HSF1 in Stress, Development, and $651 \quad$ Organismal Health. Trends Cell Biol. 27, 895-905 (2017).

652 22. Douglas, P. M. et al. Heterotypic Signals from Neural HSF-1 Separate Thermotolerance 653 from Longevity. Cell Rep. 12, 1196-1204 (2015).

654 23. Hahn, J., Hu, Z., Thiele, D. J. \& Iyer, V. R. Genome-Wide Analysis of the Biology of 655 Stress Responses through Heat Shock Transcription Factor. Mol. Cell. Biol. 24, 5249$656 \quad 5256(2004)$.

657 24. Pincus, D. et al. Genetic and epigenetic determinants establish a continuum of Hsf1 658 occupancy and activity across the yeast genome. Mol. Biol. Cell 29, 3168-3182 (2018).

659 25. Clos, J., Rabindran, S., Wisniewski, J. \& Wu, C. Induction temperature of human heat 660 shock factor is reprogrammed in a Drosophila cell environment. Nature 364, 252-5 (1993).

662 26. Hentze, N., Breton, L. Le, Wiesner, J., Kempf, G. \& Mayer, M. P. Molecular mechanism 663 of thermosensory function of human heat shock transcription factor Hsfl. eLife 5, e11576 
(2016).

665 27. Soncin, F. et al. Transcriptional activity and DNA binding of heat shock factor-1 involve 666 phosphorylation on threonine 142 by CK2. Biochem. Biophys. Res. Commun. 303, 700$667 \quad 706(2003)$.

668 28. Hashikawa, N., Yamamoto, N. \& Sakurai, H. Different mechanisms are involved in the 669 transcriptional activation by yeast heat shock transcription factor through two different types of heat shock elements. J. Biol. Chem. 282, 10333-10340 (2007).

671 29. Satyal, S. H., Chen, D., Fox, S. G., Kramer, J. M. \& Morimoto, R. I. Negative regulation 672 of the heat shock transcriptional response by HSBP1. Genes Dev. 12, 1962-1974 (1998).

673 30. Tan, K. et al. Mitochondrial SSBP1 protects cells from proteotoxic stresses by 674 potentiating stress-induced HSF1 transcriptional activity. Nat. Commun. 6, 1-15 (2015).

675 31. Neef, D. W., Jaeger, A. M. \& Thiele, D. J. Genetic selection for constitutively trimerized 676 human hsf1 mutants identifies a role for coiled-coil motifs in DNA binding. G3 Genes, 677 Genomes, Genet. 3, 1315-1324 (2013).

678 32. Takemori, Y. et al. Mutational analysis of human heat-shock transcription factor 1 reveals 679 a regulatory role for oligomerization in DNA-binding specificity. Biochem. J. 424, 253$680261(2009)$.

681 33. Sorger, P. K. \& Nelson, H. C. M. Trimerization of a yeast transcriptional activator via a $682 \quad$ coiled-coil motif. Cell 59, 807-813 (1989).

683 34. Zuo, J., Baler, R., Dahl, G. \& Voellmy, R. Activation of the DNA-binding ability of 
684 human heat shock transcription factor 1 may involve the transition from an intramolecular

685 to an intermolecular triple-stranded coiled-coil structure. Mol. Cell. Biol. 14, 7557-7568

$686 \quad(1994)$.

687 35. Giardina, C. \& Lis, J. T. Dynamic protein-DNA architecture of a yeast heat shock

$688 \quad$ promoter. Mol. Cell. Biol. 15, 2737-2744 (1995).

689 36. Crick, F. H. C. The packing of $\alpha$-helices: simple coiled-coils. Acta Crystallogr. 6, 689-

$690697(1953)$.

691 37. McLachlan, A. D. \& Stewart, M. Tropomyosin coiled-coil interactions: Evidence for an 692 unstaggered structure. J. Mol. Biol. 98, 293-304 (1975).

693 38. Clos, J. et al. Molecular cloning and expression of a hexameric Drosophila heat shock 694 factor subject to negative regulation. Cell 63, 1085-1097 (1990).

695 39. Peteranderl, R. \& Nelson, H. C. M. Trimerization of the Heat Shock Transcription Factor 696 by a Triple-Stranded $\alpha$-Helical Coiled-Coil. Biochemistry 31, 12272-12276 (1992).

697 40. Xu, W. et al. The influence of the mating type on virulence of Mucor irregularis. Sci. Rep. $698 \quad 7,1-12(2017)$.

699 41. Akaza, N. et al. Malassezia globosa tends to grow actively in summer conditions more 700 than other cutaneous Malassezia species. J. Dermatol. 39, 613-616 (2012).

701 42. Vishniac, H. S. \& Hempfling, W. P. Cryptococcus vishniacii sp. nov., an Antarctic Yeast. 702 Int. J. Syst. Bacteriol. 29, 153-158 (1979).

703 43. van den Brink, J., Facun, K., de Vries, M. \& Stielow, J. B. Thermophilic growth and 
enzymatic thermostability are polyphyletic traits within Chaetomiaceae. Fungal Biol. 119, $1255-1266$ (2015).

44. Wang, H., Mayhew, D., Chen, X., Johnston, M. \& Mitra, R. D. Calling Cards enable multiplexed identification of the genomic targets of DNA-binding proteins. Genome Res. 21, 748-755 (2011).

45. Zhou, W., Dorrity, M. W., Bubb, K. L., Queitsch, C. \& Fields, S. Binding and regulation of transcription by yeast Ste12 variants to drive mating and invasion phenotypes. Genetics 214, 397-407 (2020).

46. Yamamoto, A., Mizukami, Y. \& Sakurai, H. Identification of a novel class of target genes and a novel type of binding sequence of heat shock transcription factor in Saccharomyces cerevisiae. J. Biol. Chem. 280, 11911-11919 (2005).

715 47. Palii, C. G. et al. Differential genomic targeting of the transcription factor TAL1 in alternate haematopoietic lineages. EMBO J. 30, 494-509 (2011).

717 48. Li, C. et al. Genome-wide characterization of cis-acting DNA targets reveals the transcriptional regulatory framework of Opaque2 in maize. Plant Cell 27, 532-545 (2015).

49. Wang, S. et al. Target analysis by integration of transcriptome and ChIP-seq data with BETA. Nat. Protoc. 8, 2502 (2013).

722 50. Opulente, D. A. et al. Factors driving metabolic diversity in the budding yeast subphylum. BMC Biol. 16, 1-15 (2018). 
724 51. Kurtzman, C., Fell, J. W. \& Boekhout, T. The yeasts: a taxonomic study. (Elsevier, 2011).

725 52. Nover, L. et al. Arabidopsis and the heat stress transcription factor world: How many heat 726 stress transcription factors do we need? Cell Stress Chaperones 6, 177-189 (2001).

727 53. Chen, Y., Barlev, N. a, Westergaard, O. \& Jakobsen, B. K. Identification of the C-terminal 728 activator domain in yeast heat shock factor: independent control of transient and sustained 729 transcriptional activity. EMBO J. 12, 5007-18 (1993).

730 54. Jakobsen, B. K. \& Pelham, H. R. A conserved heptapeptide restrains the activity of the 731 yeast heat shock transcription factor. EMBO J. 10, 369-375 (1991).

732 55. Puustinen, M. C. \& Sistonen, L. Molecular Mechanisms of Heat Shock Factors in Cancer. 733 Cells 9, 1-20(2020).

734 56. Hashikawa, N., Mizukami, Y., Imazu, H. \& Sakurai, H. Mutated yeast heat shock 735 transcription factor activates transcription independently of hyperphosphorylation. J. Biol. $736 \quad$ Chem. 281, 3936-3942 (2006).

737 57. Jolma, A. et al. DNA-dependent formation of transcription factor pairs alters their binding specificity. Nature 527, 384-388 (2015).

739 58. Rohs, R., West, S. M., Sosinsky, A., Liu, P. \& Mann, R. S. The role of DNA shape in 740 protein-DNA recognition. Nature 461, (2009).

741 59. Ali, M. H. \& Imperiali, B. Protein oligomerization: How and why. Bioorganic Med. $742 \quad$ Chem. 13, 5013-5020(2005).

743 60. Ulmasov, T., Hagen, G. \& Guilfoyle, T. J. Dimerization and DNA binding of auxin 
response factors. Plant J. 19, 309-319 (1999).

745 61. Dorrity, M. W., Cuperus, J. T., Carlisle, J. A., Fields, S. \& Queitsch, C. Preferences in a trait decision determined by transcription factor variants. Proc. Natl. Acad. Sci. 115,

62. Mumberg, D., Müller, R. \& Funk, M. Yeast vectors for the controlled expression of E7997-E8006 (2018).

750

63. Mnaimneh, S. et al. Exploration of essential gene functions via titratable promoter alleles. Cell 118, 31-44 (2004).

64. Edgar, R. C. MUSCLE: Multiple sequence alignment with high accuracy and high throughput. Nucleic Acids Res. 32, 1792-1797 (2004).

754 65. Crooks, G. E., Hon, G., Chandonia, J.-M. \& Brenner, S. E. WebLogo: a sequence logo generator. Genome Res. 14, 1188-1190 (2004).

66. Simonetti, F. L., Teppa, E., Chernomoretz, A., Nielsen, M. \& Marino Buslje, C. MISTIC: mutual information server to infer coevolution. Nucleic Acids Res. 41, W8--W14 (2013). carrier DNA/polyethylene glycol method. Methods Enzymol. 350, 87-96 (2002). 
Res. 43, W39--W49 (2015).

765 70. Teixeira, M. C. et al. YEASTRACT: an upgraded database for the analysis of

766 transcription regulatory networks in Saccharomyces cerevisiae. Nucleic Acids Res. 46,

767 D348--D353 (2018).

768 71. Reimand, J. et al. g: Profiler - a web server for functional interpretation of gene lists

769 (2016 update). Nucleic Acids Res. 44, W83--W89 (2016).

$77072 . \quad$ Cuperus, J. T., Lo, R. S., Shumaker, L., Proctor, J. \& Fields, S. A tetO Toolkit to Alter

771 Expression of Genes in Saccharomyces cerevisiae. ACS Synth. Biol. 4, 842-852 (2015).

772 73. Supek, F., Bošnjak, M., Škunca, N. \& Šmuc, T. Revigo summarizes and visualizes long

$773 \quad$ lists of gene ontology terms. PLoS One 6, (2011).

774 74. Storici, F. \& Resnick, M. A. The delitto perfetto approach to in vivo site-directed

775 mutagenesis and chromosome rearrangements with synthetic oligonucleotides in yeast.

$776 \quad$ Methods Enzymol. 409, 329-345 (2006).

777 75. Åkerfelt, M., Morimoto, R. I. \& Sistonen, L. Heat shock factors: Integrators of cell stress, 778 development and lifespan. Nat. Rev. Mol. Cell Biol. 11, 545-555 (2010).

779 76. Littlefield, O. \& Nelson, H. C. M. A new use for the'wing' of the'winged'helix-turn-helix

780 motif in the HSF-DNA cocrystal. Nat. Struct. Mol. Biol. 6, 464-470 (1999).

781 77. Cicero, M. P. et al. The wing in yeast heat shock transcription factor (HSF) DNA-binding 782 domain is required for full activity. Nucleic Acids Res. 29, 1715-1723 (2001). 


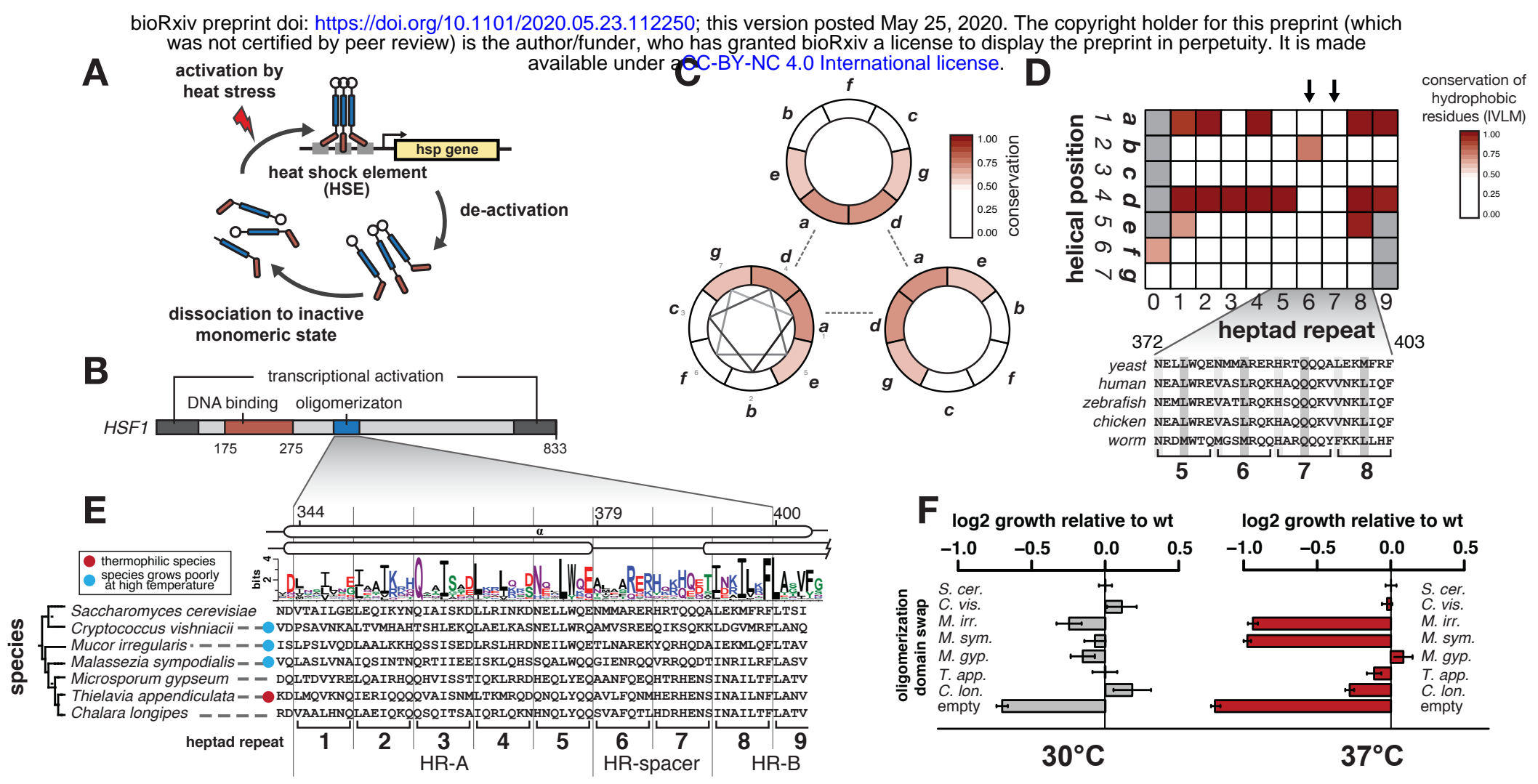

Figure 1. 
Figure 1. The Hsf1 oligomerization domain contributes to temperature-specific fitness and exhibits a conserved interruption of hydrophobic periodicity. (A) The standard model of induced Hsf1 activity involves conversion from an inactive monomer to a hyper-phosphorylated trimer upon stress exposure. Trimer binding to heat shock elements (HSEs) leads to upregulation of stress response genes such as heat shock proteins (HSP). The cycle is attenuated through multiple mechanisms, including interaction with heat shock proteins and acetylation. ${ }^{75}$ (B) The S. cerevisiae Hsf1 protein contains $\mathrm{N}$ - and $\mathrm{C}$-terminal activation domains along with highly conserved DNA binding and oligomerization domains. (C) The oligomerization domain of Hsf1 has an a-helical structure (one turn $=3.6$ residues). A helical-wheel representation in which each position (labeled abcdefg) in the helix is shown, with dotted lines indicating the predicted hydrophobic interactions in the trimer between residues $a$ and $d$. Color indicates the degree of mean conservation of sites in the oligomerization domain grouped by helix position among 1229 sequenced fungal species. (D) The conservation of hydrophobic residues (Ile, Val, Leu, Met) in fungal species is broken down by helical position (a-g) and by heptad (1-9), showing conservation of hydrophobicity at residues $a$ and $d$ with the exception of heptads 6 and 7. The sequence of this equivalent region in HSFs from human, zebrafish, chicken, and $C$. elegans is shown below. (E) Six fungal species with Hsf1 oligomerization domains of either high predicted trimer propensity (red dot) or low predicted trimer propensity (blue dot) are shown below a sequence logo plot of this domain generated from fungal species. Two different predictions of a-helical regions are shown above: separation of the domain into two helixes (HR-A and HR-B) ${ }^{33}$ or one continuous a-helix. (F) Amino acids 342-403 of the S. cerevisiae Hsf1 were replaced with the oligomerization domain of one of the six fungal species shown in (E).

Endogenous S. cerevisiae HSF1 was repressed and rescued with one of these chimeric sequences, wild-type $S$. cerevisiae HSF1, or an empty vector control plasmid. Growth at $30^{\circ} \mathrm{C}$ or $37^{\circ} \mathrm{C}$ was assayed in a plate reader overnight. Maximum slope of the resulting growth curves relative to the wild-type rescue is presented (error bars represent standard error of the mean for eight technical replicate wells). 
bioRxiv preprint doi: https://doi.org/10.1101/2020.05.23.112250; this version posted May/25, 2020. The Gopyright holder for this preprint (which

A

was not certified by peer review) is the author/funder, who has granted bioRxiv a license to display thespepeprint in perpetuity. It is made growth rate

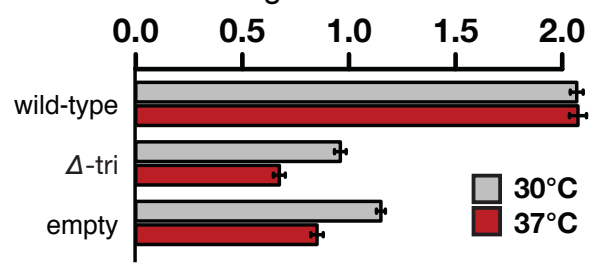

avarable

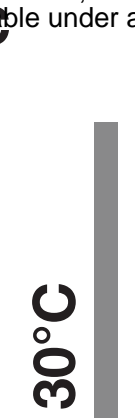

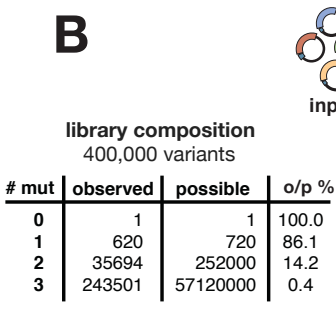

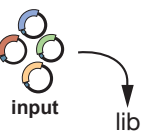

365

wild-type sequence

400

L LR INKDNELIT QENMMARERHRT QQQALEKMFRFI
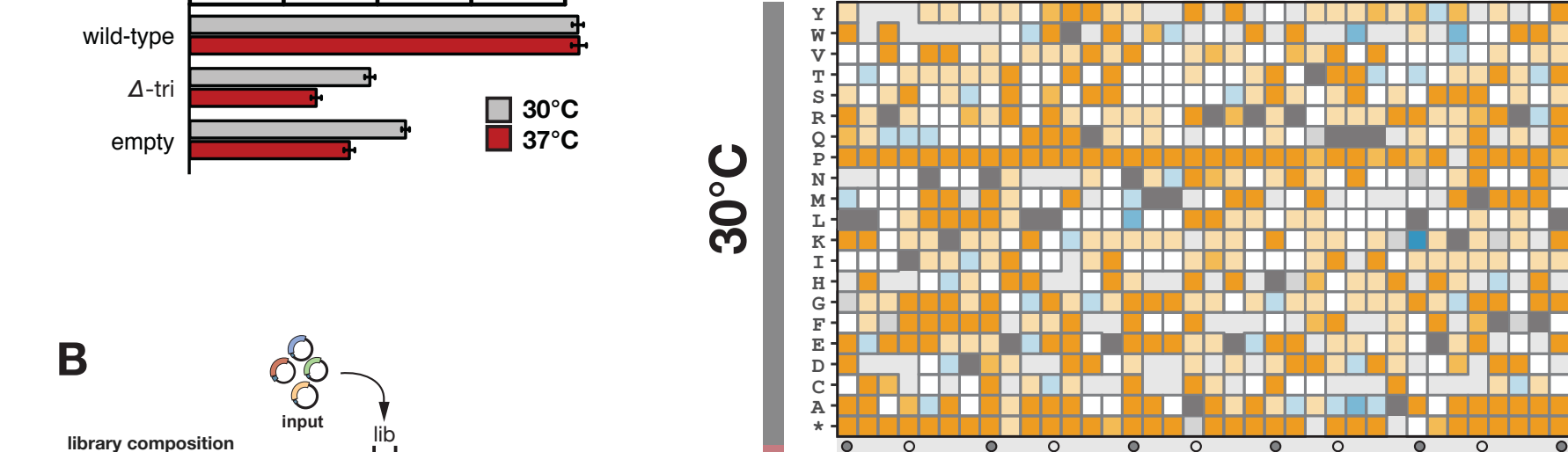

\section{relative \\ fitness (log2)}

\begin{tabular}{c|c} 
& $>+3$ \\
& +2 \\
$\square$ & +1 \\
0 \\
$\square$ \\
$-\square$ \\
$-\square$ \\
$-\square-3$
\end{tabular}
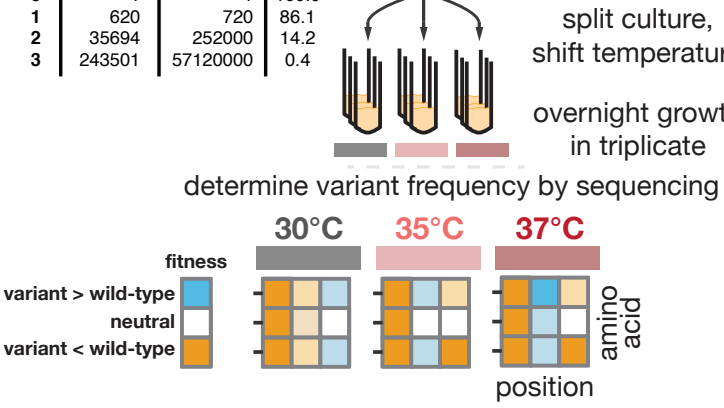

shift temperature

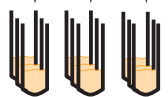

overnight growth

in triplicate

determine variant frequency by sequencing

D

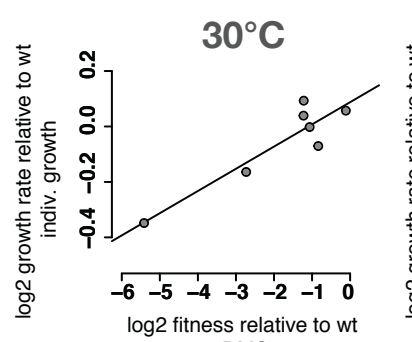

DMS

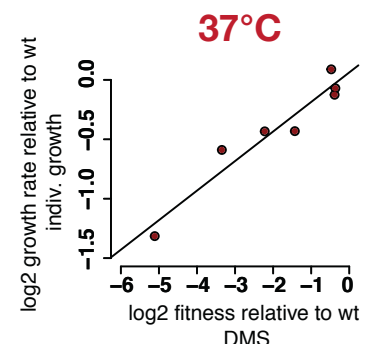

DMS

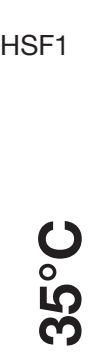

0
$\stackrel{0}{\infty}$

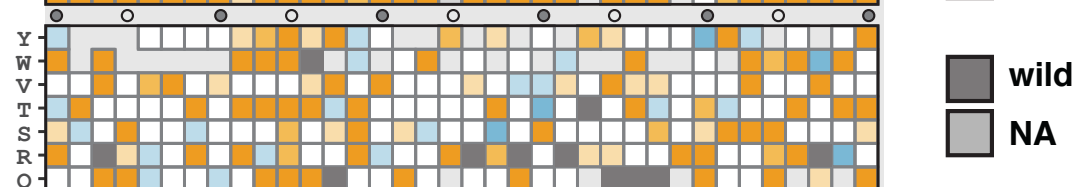

helix phase

RINKDNELLWQENMMARERHRTQQQALEKMFRFL

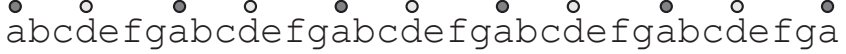

Figure 2. 
Figure 2. A screen of over 400,000 variants in the Hsf1 oligomerization domain reveals positional fitness effects under basal and heat stress conditions. (A) Yeast endogenous HSF1 was repressed with anhydrotetracycline (ATc) and rescued with plasmid-based expression of either a wild-type HSF1, an HSF1 clone with the oligomerization domain deleted (residues 342-403 deleted), or an empty vector control plasmid. Growth of these strains was assayed overnight in a plate reader held at either $30^{\circ} \mathrm{C}$ or $37^{\circ} \mathrm{C}$. Values on the $y$-axis represent maximum slope of the resulting growth curves. (B) A deep mutational scan (DMS) was conducted using a library of 400,000 variants in residues $365-400$ of the oligomerization domain of a plasmid-based $S$. cerevisiae HSF1. This library contained $86 \%$ of the possible single residue changes and $14 \%$ of all possible double residue changes. The library was transformed into an $S$. cerevisiae strain with tet-off HSF1 so that endogenous HSF1 could be repressed with ATc. The culture was split into three replicates at each of three temperatures $\left(30^{\circ} \mathrm{C}\right.$ for 14.5 hours, $35^{\circ} \mathrm{C}$ for 14.5 hours, or $37^{\circ} \mathrm{C}$ for 17.5 hours), after which plasmid libraries were extracted and sequenced for frequency. A simulated result output format is shown in (B), wherein a decrease in variant HSF1 relative to wild type (orange) or an increase in variant HSF1 frequency relative to wild type (blue) is presented in grid form of residue position by amino acid change, for each of the three temperatures. (C) The relative fitness scores of yeast expressing each single mutation variant in the DMS library are presented as a heat map for growth at $30^{\circ} \mathrm{C}, 35^{\circ} \mathrm{C}$, and $37^{\circ} \mathrm{C}$. Relative fitness determined as variant $(\log 2(37 /$ input $))$ - wild-type $(\log 2(37 /$ input $))$, where the input is the composition of the library pre-yeast transformation. Wild-type amino acid sequence is show above and below the plots, with circles marking residues at helix phase positions $a$ and $d$. The region of the oligomerization domain covered in the mutagenesis is diagramed above (highlighted in purple). Variant L393K showed an enrichment score of $>6.6$ for all temperatures, making it more likely a sequencing error than a true enrichment. Indeed, L393K failed to show a growth advantage in validation studies (data not shown). The L393K mutation was therefore removed from subsequent analyses. (D) Seven additional HSF1 single mutants spanning a range of fitness effects were chosen from the library and independently cloned (variants A382P, L375S, I368R, Q389K, N379I, I368L, A392N). These variants were assessed for growth in a plate reader assay as in (A). Their growth relative to wild type is plotted here against their fitness scores in the DMS. 
bioRxiv preprint doi: https://doi.org/10.1101/2020.05.23.112250; this version posted May 25, 2020. The copyright holder for this preprint (which was not certified by peer review) is the author/funder, who has granted bioRxiv a license to display the preprint in perpetuity. It is made

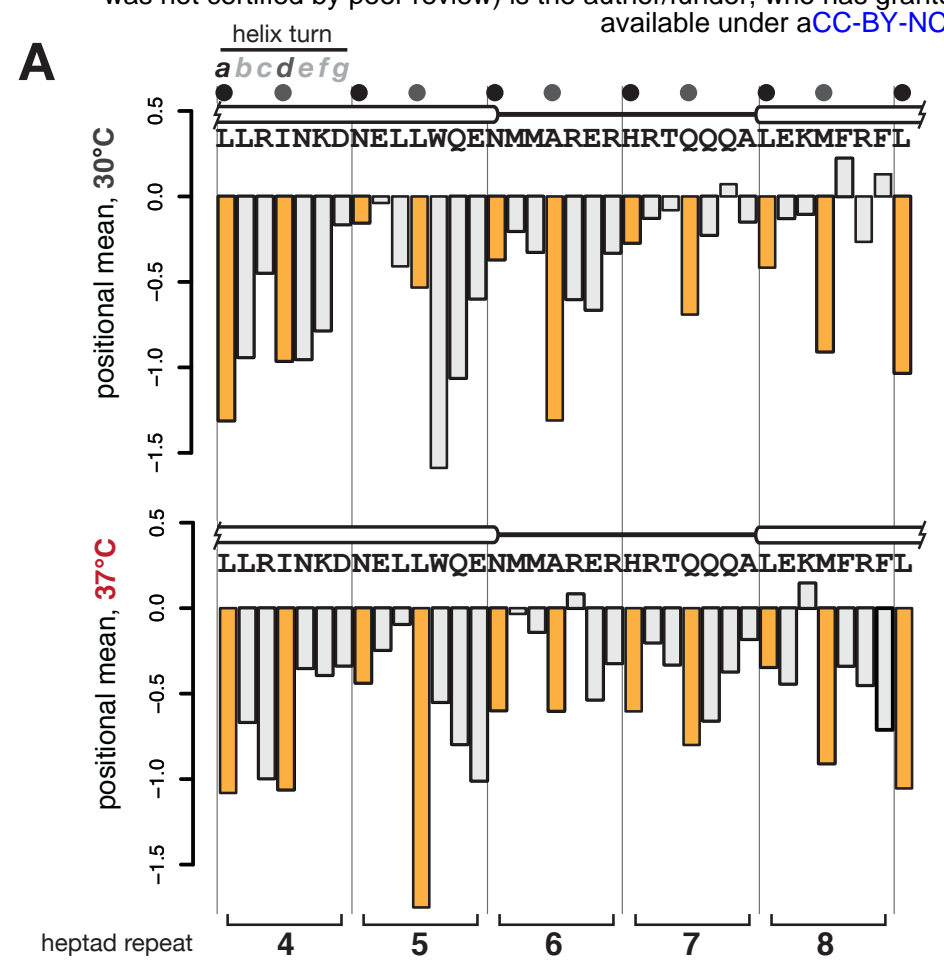

B mean fitness effect of small and
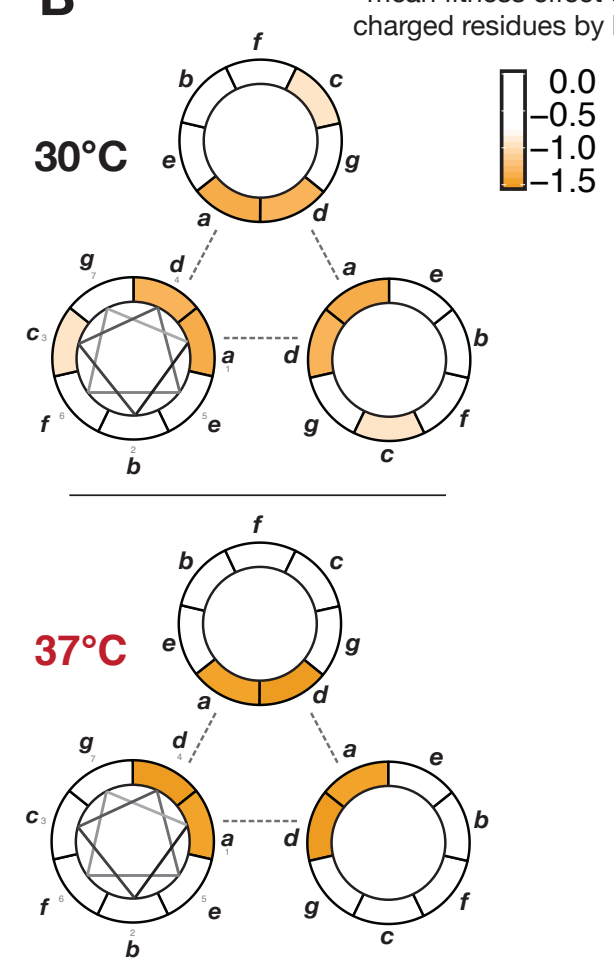

C

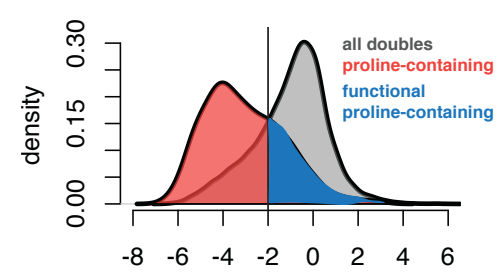

$37^{\circ} \mathrm{C}$ growth relative to wt

E
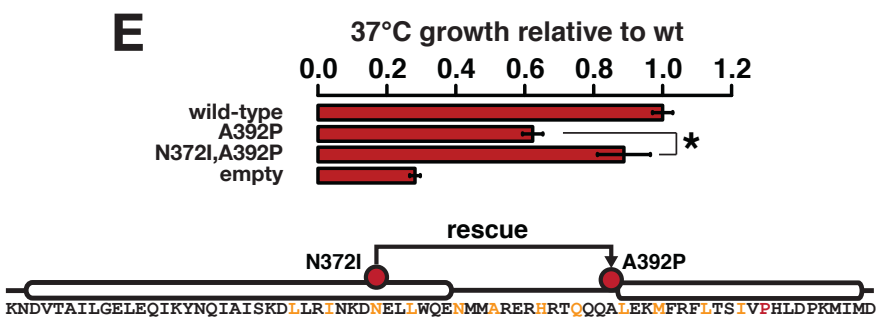

D

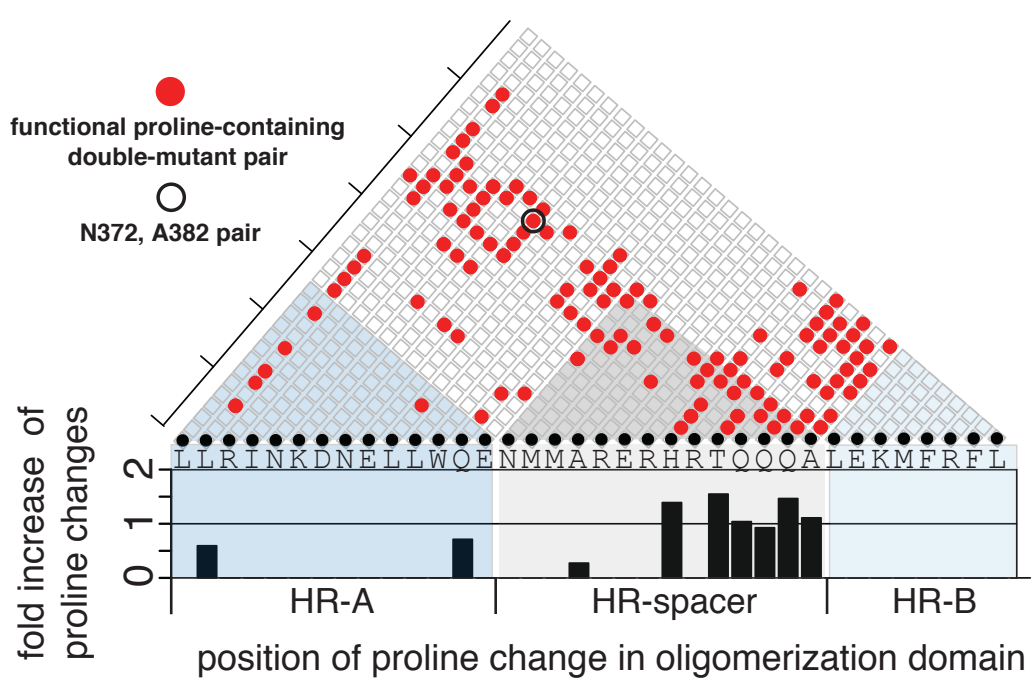

Figure 3. 
Figure 3. Positional effects of Hsf1 variants reveal differing mutational sensitivities for oligomerization domain in the presence and absence of stress. (A) Barplots showing the mean (log2) of fitness scores of all single amino acid changes at each position in the mutagenized region of oligomerization domain under basal (top) and heat-stress (bottom) conditions. Positions in the helical core ( $a$ and $d$ ) are colored in orange, and helical positions are indicated above. Boundaries of heptad repeats are shown with vertical lines. (B) Helix loop plots showing the mean effect of single amino changes to small or charged residues (K,R,D,E,S,G), grouped by each of the seven helix positions $a-g$. More deleterious effects are shown in shades of orange. (C) Density histograms show the distribution of $37^{\circ} \mathrm{C}$ fitness scores for all double mutants (gray) or double mutants that contain at least one proline (red). While the proline-containing distribution is strongly shifted in the negative direction, consistent with their unanimously deleterious effect, a fraction of these double mutants are less deleterious, and their overlap with the non-proline-containing distribution is shown in blue. (D) A pair-wise mutational plot indicates the positions of the pairs of mutations in the less-deleterious group of proline-containing double mutants (blue population in panel $\mathrm{C}$ ). Above the wildtype sequence, each box represents a possible site pair; red circles indicate pairs found in the set of less-deleterious proline doubles. While this triangular plot does not indicate which of the two sites contributed the deleterious proline, the barplot below indicates the relative frequency of prolines at each individual site in the less-deleterious mutant set. Despite the diversity of positions among the site pairs, the contributing prolines appear highly enriched within the HR-spacer. The absence of other sites containing proline (no bar) indicates that variants at these sites were completely absent in the output population. (E) Growth rate experiments (as in Fig. 2A) independently confirm a proline double mutant identified in the screen whose deleterious effects could be mitigated by a second mutation in HR-A. Growth relative to wild type is compared between strains expressing the proline single mutant (A392P), the double mutant (A392P, N372I), and a negative control with no Hsf1 rescue (empty). A schematic of the location of each mutated position is shown below, with residues at $a$ and $d$ helix positions marked in orange. (error bars represent standard error of the mean for eight technical replicate wells, * $t$-test $p$-value $<0.05$ ) 
bioRxiv preprint doi: https://doi.org/10.1101/2020.05.23.112250; this version posted May 25 2020. The copyright holder for this preprint (which

A

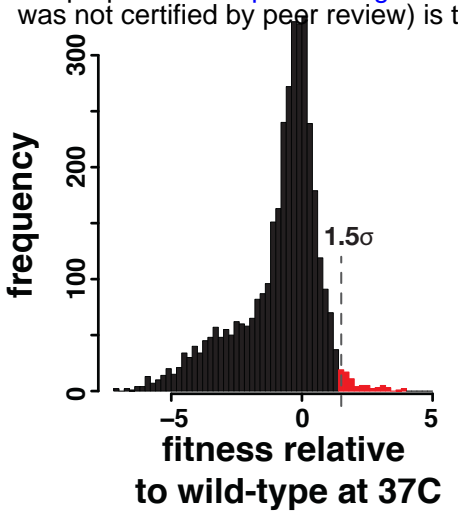

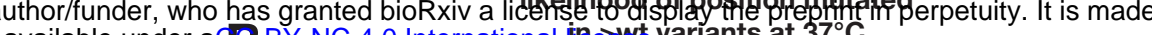

available under ad 8 -BY-NC 4.0 International ine exks variants at $37^{\circ} \mathrm{C}$

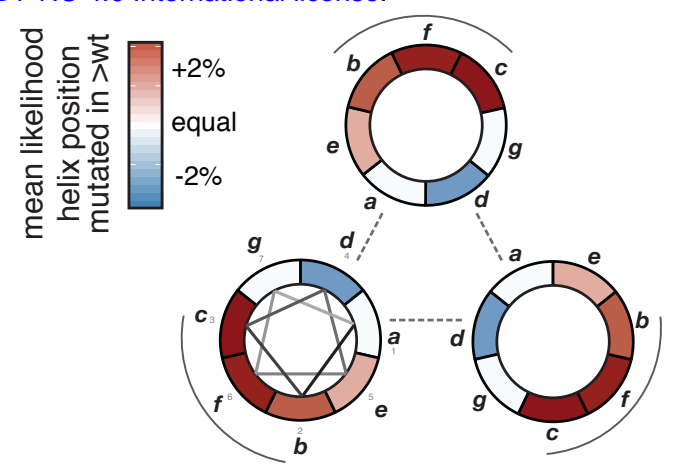

C

$30^{\circ} \mathrm{C}$

$37^{\circ} \mathrm{C}$

$\log 2$ growth relative to $\mathrm{wt} \quad \log 2$ growth relative to $\mathrm{wt}$

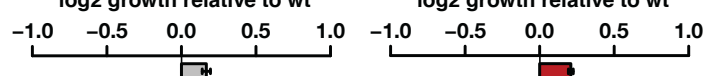
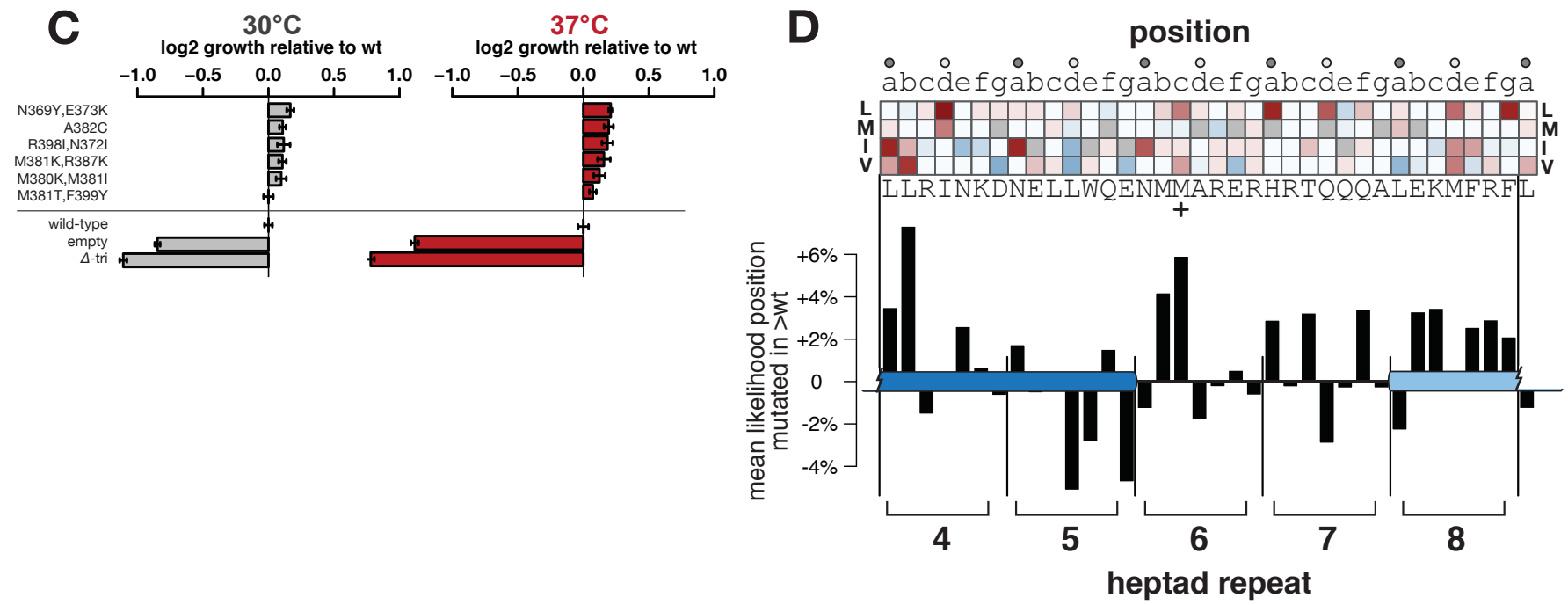

Figure 4. 
Figure 4. Exceptional Hsf1 variants show increased fitness under stress. (A) The relative fitness scores at $37^{\circ} \mathrm{C}$ for all variants are presented as a histogram. Exceptional variants were identified as those with fitness scores beyond the indicated 1.5 standard deviation cutoffs (red bars) in terms of fitness relative to wild type. (B) Variants with fitness benefits are enriched at outer helix face positions. Helix position plots reveal the frequency of mutation in this subset of variants, grouped by helix position; red shading represents an enrichment in mutation at that helix position and blue represents a depletion. (C) A set of variants chosen for their growth rate relative to wild-type in the DMS were individually validated (as in Fig. $1 \mathrm{~F}$ ) at basal (left) and heat-shock (right) temperatures. (D) Among the subset of exceptional variants, enrichment for specific substitutions (hydrophobic residues I,V,L,M, y-axis) at each position in the oligomerization domain (x-axis) was analyzed and presented as a heat map (red represents enrichment, blue represents depletion) (See Fig. S10A for full heat map). Helix positions are indicated above the map. The mean enrichment per-site is shown in the barplot below; the most enriched site is at position $b$ in HR-A, and the second most enriched site is at position $c$ in the HR-spacer (heptad boundaries shown as vertical bars). 
bioRxiv preprint doi: https://doi.org/10.1101/2020.05.23.112250; this version posted May 25,2020 . The copyright holder for this preprint (which
was not certified by peer reviev/Ris the author/funder, who has granted bioR a license to display the preprint in Perpetuity. It is made
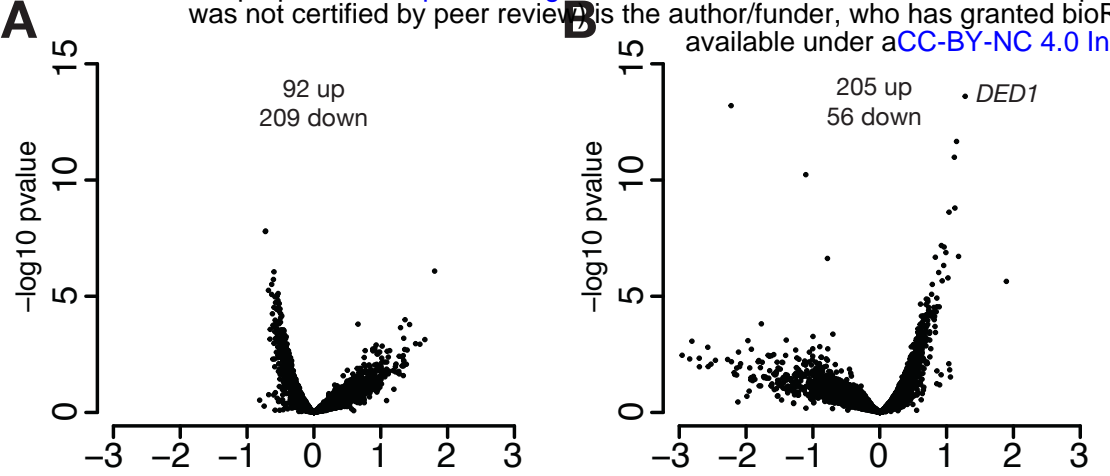

log2 fold change exceptional vs wt, $30^{\circ} \mathrm{C}$

log2 fold change exceptional vs wt, $37^{\circ} \mathrm{C}$

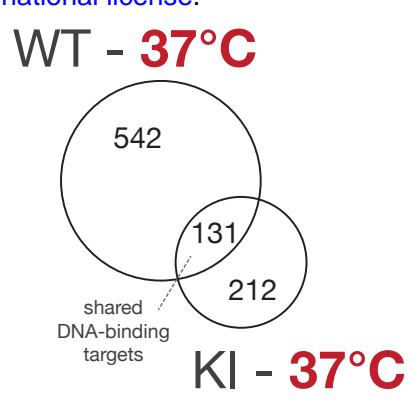

E

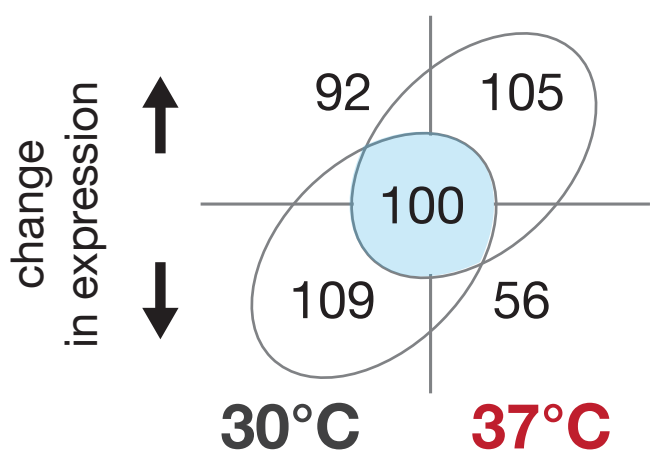

F

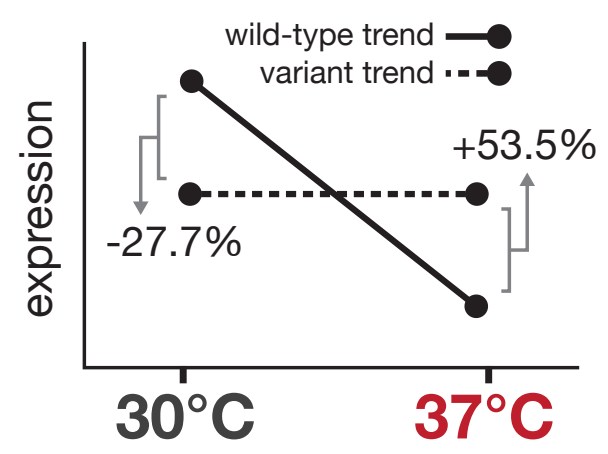

100 shared genes
HSE type

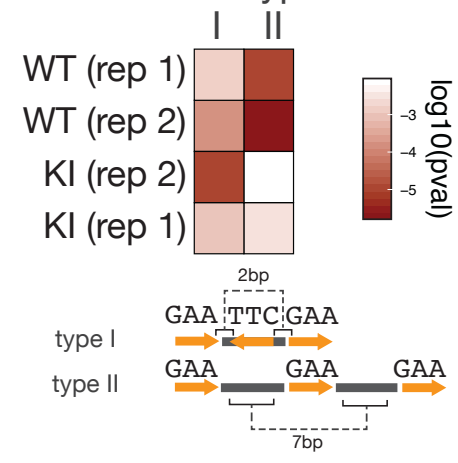

Figure 5. 
Figure 5. Hsf1 variants with enhanced fitness under heat stress show alterations in DNA binding and loss of temperature-dependent regulation of a set of genes. RNA-sequencing was performed on yeast with endogenous HSF1 repressed and rescued with a plasmid expressing wild type (two independent clones) or one of two HSF1 variants (N369Y, E373K and M380K, M381I, data treated as replicates in analysis). Results are presented for 120 minutes of growth. (A) For a p-value cutoff of $0.01,92$ genes were differentially upregulated in variants compared to wild type at $30^{\circ} \mathrm{C}$ and 209 genes were differentially downregulated. (B) At $37^{\circ} \mathrm{C}, 205$ genes were differentially upregulated in the variants compared to wild type and 56 genes differentially downregulated. (C) Hsf1 binding at $37 \mathrm{C}$ was assessed using the transposon-insertion-based method "calling card." The gene on either side of each highconfidence transposon insertion site was determined, tallying 673 total possible gene targets for wild type (WT) and 343 possible gene targets for variant M380K,M381I (KI), of which 131 genes were the same. (D) Motif enrichment for two different HSEs - Type I (GAAnnTTCnnGAA) and Type II (GAAnnnnnnnGAAnnnnnnnGAA) - was determined by AME (see methods) from calling card binding data for wild-type (WT) Hsf1 and the $\mathrm{M} 380 \mathrm{~K}, \mathrm{M} 381 \mathrm{I}$ variant $(\mathrm{KI})$ at $37^{\circ} \mathrm{C}$. Heat map values represent $\log 10$ of the $\mathrm{p}$-value motif enrichment. The $p$-values displayed have been Bonferroni corrected for multiple sequences tested. For E-values (adjusted p-values corrected for multiple motifs) see Table S5. (E) In the RNA-seq results at the 120min timepoint, 209 genes were downregulated at $30^{\circ} \mathrm{C}$ in the variants with enhanced stress resistance relative to wild type. This set overlapped by 100 genes with the 205 genes upregulated in variants relative to wild type at $37^{\circ} \mathrm{C}$. Most of the genes in this 100 gene list were targets significantly downregulated in wild type at $37^{\circ} \mathrm{C}$ compared to $30^{\circ} \mathrm{C}$. The right panel of (E) shows a schematized depiction of this general trend, in which these genes are downregulated with heat stress in wild type but show more temperature-consistent expression in variants (N369Y,E373K and M380K,M381I). The average percent difference in expression level for these genes in wild type vs. variants is overlaid on the schematic drawing. (F) Gene ontology analysis revealed categories enriched in the set of 100 genes that have lost the temperature-dependent downregulation. 
bioRxiv preprint doi: https://doi.org/10.1101/2020.05.23.112250; this version posted May 25, 2020. The copyright holder for this preprint (which was not certified by peer review) is the author/funder, who has granted bioRxiv a license to display the preprint in perpetuity. It is made

A

A $p=0.005$

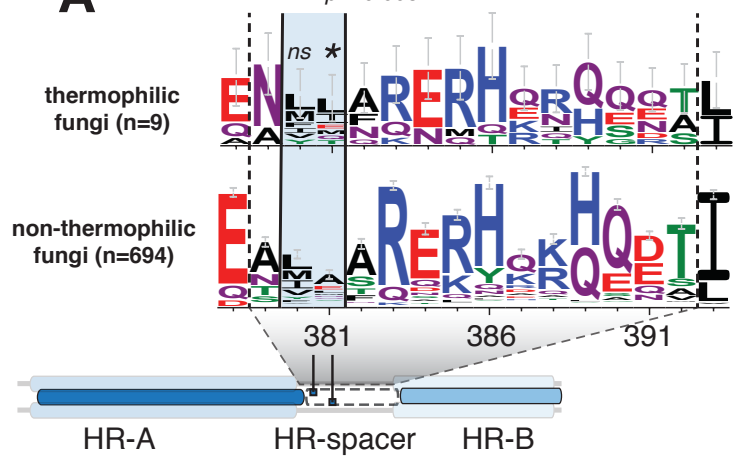

avaitable under aCC-BY-NC 4.0 International license.

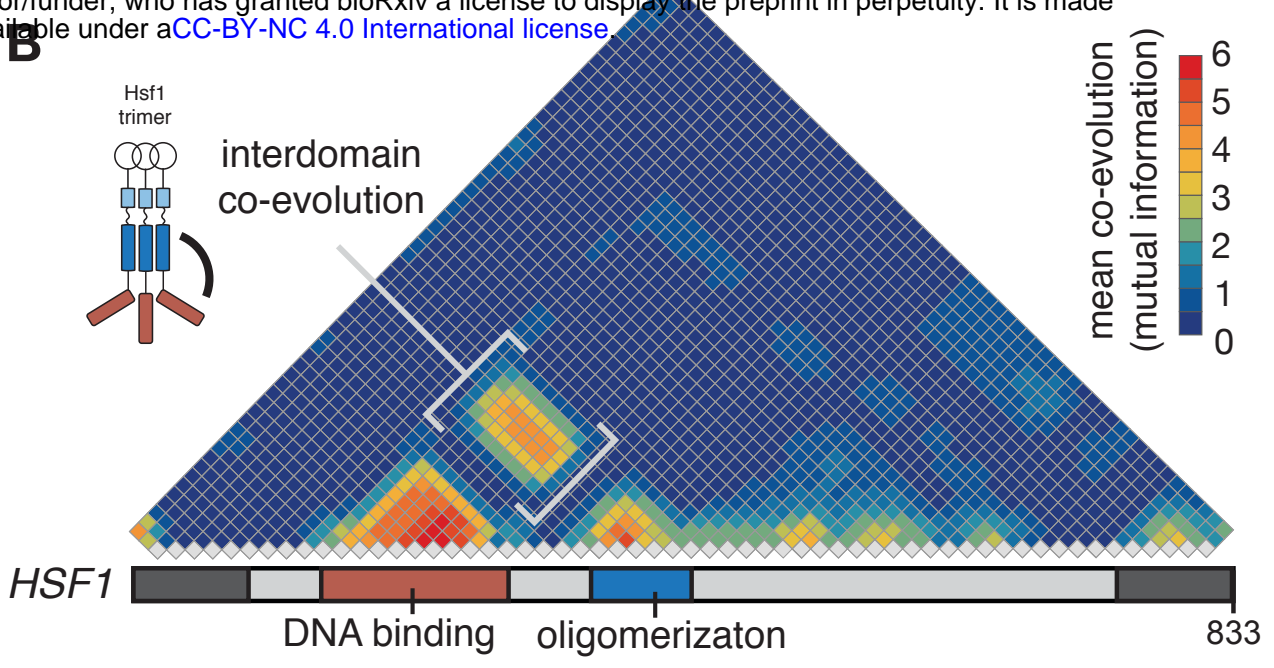

C

161

HR-A

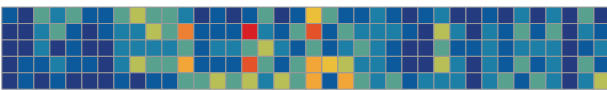

spacer

HR-B

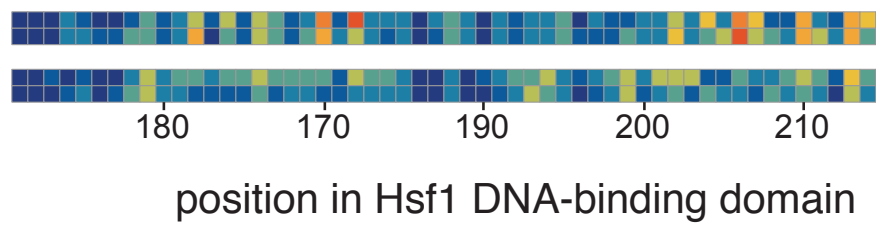

D

wild-type Hsf1 function

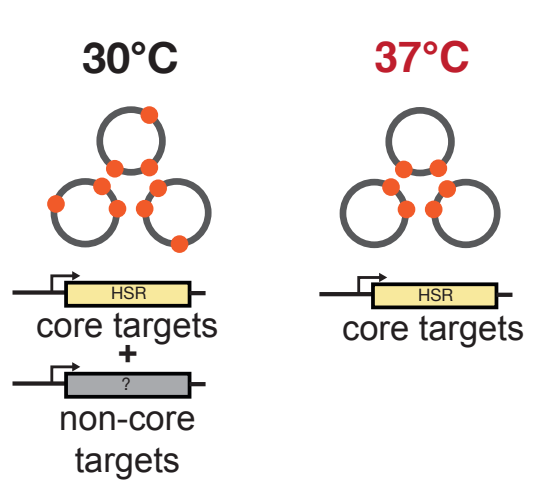

mutation-sensitive site stress-resistance

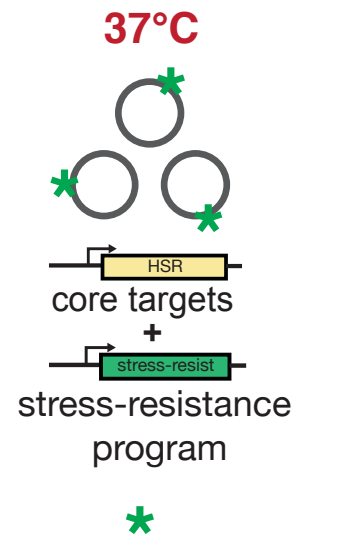

mutation conferring stress-resistance (M381I)
E

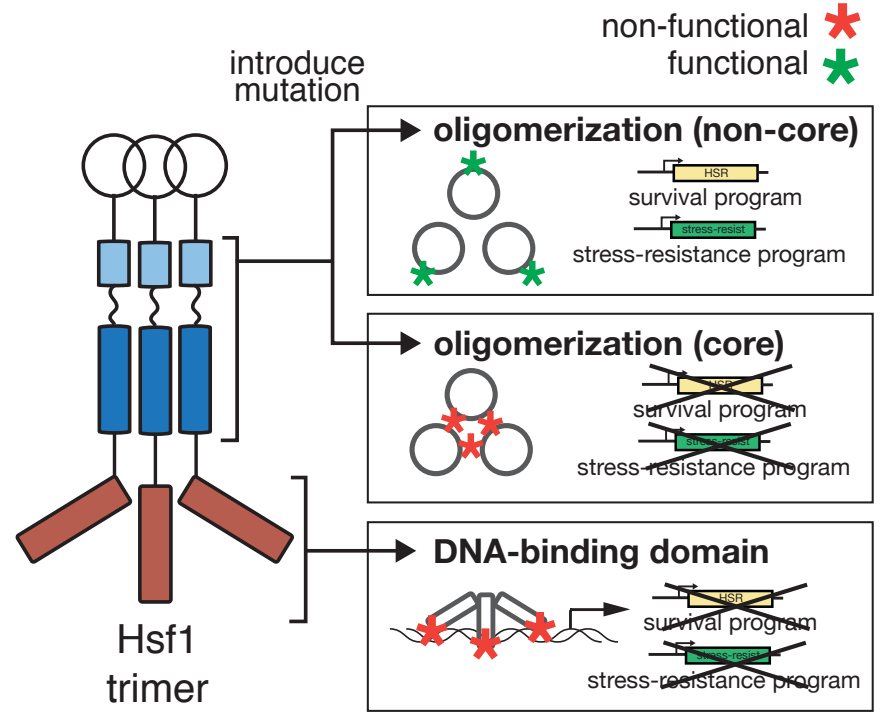

Figure 6. 
Figure 6. Analysis of natural variation in the oligomerization domain of Hsf1 reveals strong co-evolution between the oligomerization and DNA-binding domains. (A) Weblogo plots show the relative amino acid preferences within the HRspacer (bound by dotted lines) for nine thermophilic fungi with a sequenced genome. Among these thermophiles, a significant (Fisher's exact test $p=0.005$ ) preference for hydrophobic amino acids (I, V, L, M) was found at the M381 position. The preference for hydrophobic amino acids at the M380 position was not significant (ns). The position of these sites within the oligomerization domain is shown in a schematic (HR-A in dark blue, HR-spacer with dotted lines, and HR-B in light blue). (B) Intramolecular coevolution at the level of the entire Hsf1 protein was determined using pairwise mutational information between positions in the Hsf1, derived from analysis of Hsf1 protein sequences from 1200 fungal species. The $833^{\wedge} 2$ grid of all-by-all positions pairs was reduced to a $30^{\wedge} 2$ grid ( 28 aa segments of Hsf1 protein), and the mean mutual information was derived for each bin of pair-wise sites $\left(28^{\wedge} 2=784\right.$ site pairs per cell in heatmap). Binned mutual information values were smoothed with bilinear interpolation of this matrix (resolution $=1.5$ ). Higher levels of mutual information are indicated by warmer colors. Signal near the bottom of the largest triangle indicates co-evolution between proximal residues (see triangle above DNA-binding domain). Within-domain co-evolution can also be observed in the oligomerization domain. The primary betweendomain signal occurs the DNA-binding and oligomerization domains (indicated in brackets). (C) A zoom-in on all (non-binned) site pairs between the DNA-binding domain ( $x$-axis) and heptads of the oligomerization domain (y-axis). Key regions of the DNAbinding domain are indicated with black boxes along the x-axis. ${ }^{76,77}$ (D) A model schematic showing the overall pattern of mutation-sensitive sites with respect to helix position as determined by deep mutational scanning. Regardless of added temperature stress, positions in the helical core remain sensitive to mutation, while sites outside the helical core show some sensitivity in the absence of stress. The expected transcriptional targets are shown below in each case, with the heat-shock response shown in yellow gene model, and other basal targets shown in grey. In exceptional variants that activate a stress-resistance transcriptional program (green gene model), we observe an abundance of mutations on the outer helix face, away from the helical core. (E) Though exceptional variants showing stress-resistance depend on altering the wild-type transcriptional program of Hsf1, mutations in the DNA-binding domain, or in the helical core of the oligomerization domain are unlikely to retain proper targeting for the core, essential targets of Hsf1, thus leaving the outer helix face of the trimer as a viable path toward altering transcriptional targets. 
A

S. cerevisiae
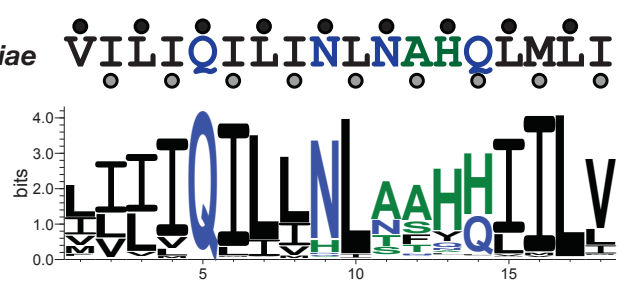

B

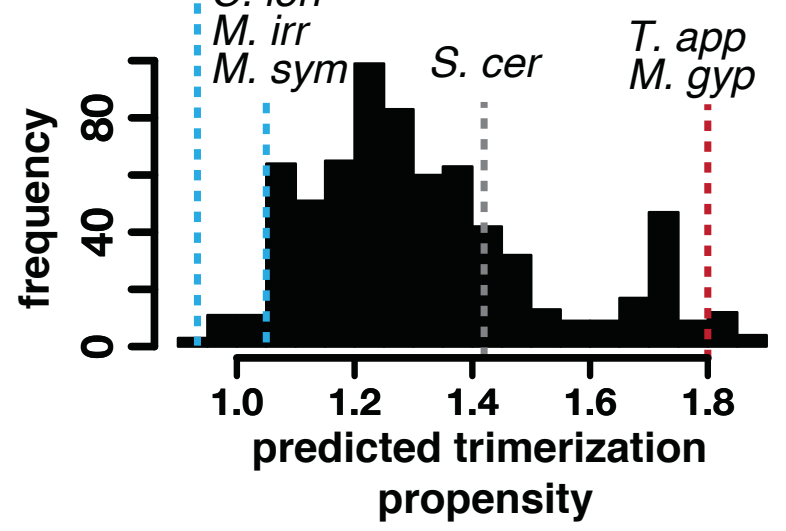

Supplementary Figure 1. Hsf1 oligomerization domain sequences across fungi conserve hydrophobic residues at a and $\mathrm{d}$ positions as well as display differences in predicted trimer propensity. (A) Logo plot showing the frequency of amino acids at the helical positions a (black dot) and d (grey dot) within the Hsf1 trimerization domain. The wild-type S. cerevisiae sequence at these a and d positions is shown above for reference (V344 to 1403). (B) Histogram showing LOGICOIL model outputs of trimerization propensity for oligomerization domains of 1229 sequenced fungi. Higher values indicate higher probability of that sequence conferring a trimeric coiled-coil. The prediction score for specific species tested in oligomerization domain swap experiments (Fig 1F) are indicated, along with the prediction for wild-type $S$. cerevisiae. 
bioRxiv preprint doi: https://doi.org/10.1101/2020.05.23.112250; this version posted May 25, 2020. The copyright holder for this preprint (which was not certified by peer review) is the author/funder, who has granted bioRxiv a license to display the preprint in perpetuity. It is made

A

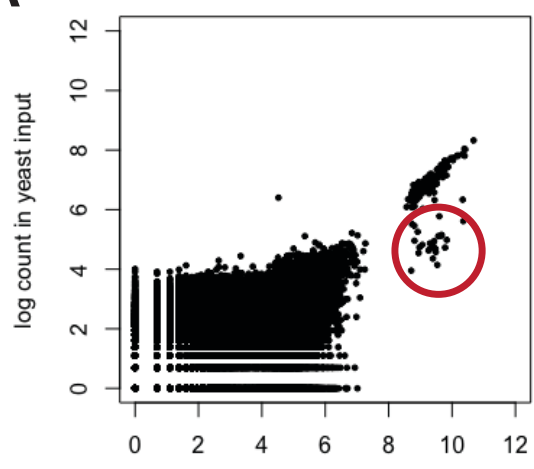

log count in plasmid pool arailable under aCC-BY-NC 4.0 International license.

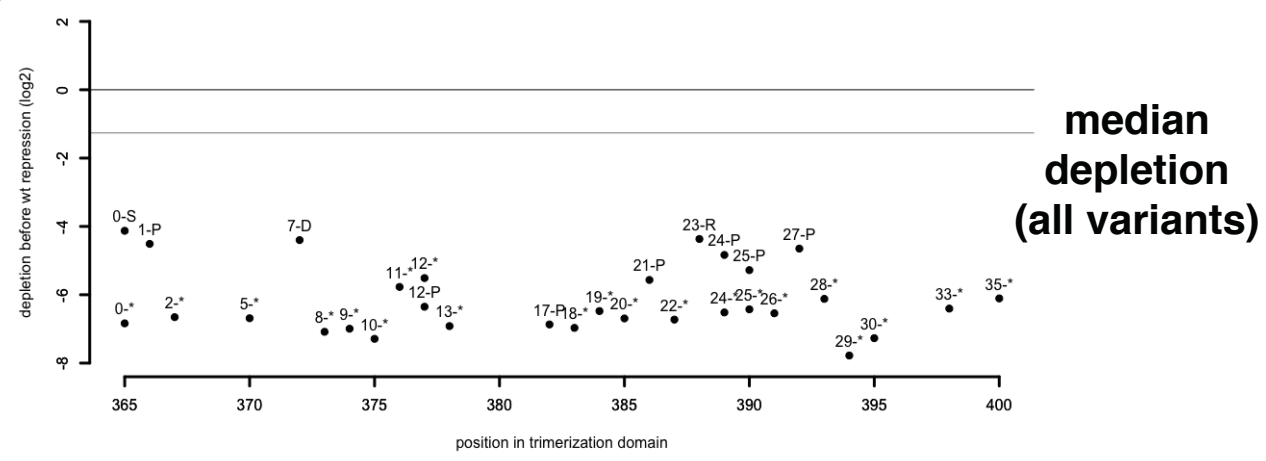

Supplementary Figure 2. Hsf1 variants depleted in the population prior to repression of endogenous Hsf1 may represent dominant negative missense variants. (A) Scatterplot showing the frequency of each variant in the plasmid pool before yeast transformation (x-axis) and after yeast transformation and outgrowth without tet-induced repression of native HSF1 (y-axis). Variants showing significant depletion (circled in red) while native HSF1 is expressed presumably act through dominant negative inhibition of Hsf1. (B) Log2 fitness scores relative to wild type frequency during outgrowth of circled variants from $(A)$ are plotted against their residue position in the Hsf1 trimerization domain. Gray horizontal line represents the median depletion of the rest of the library. The large majority of these variants represent nonsense mutations. 
bioRxiv preprint doi: https://doi.org/10.1101/2020.05.23.112250; this version posted May 25, 2020. The copyright holder for this preprint (which

was not certified by peer review) is the author/funder, who has granted bioRxiv a license to display the preprint in perpetuity. It is made available under aCC-BY-NC 4.0 International license.

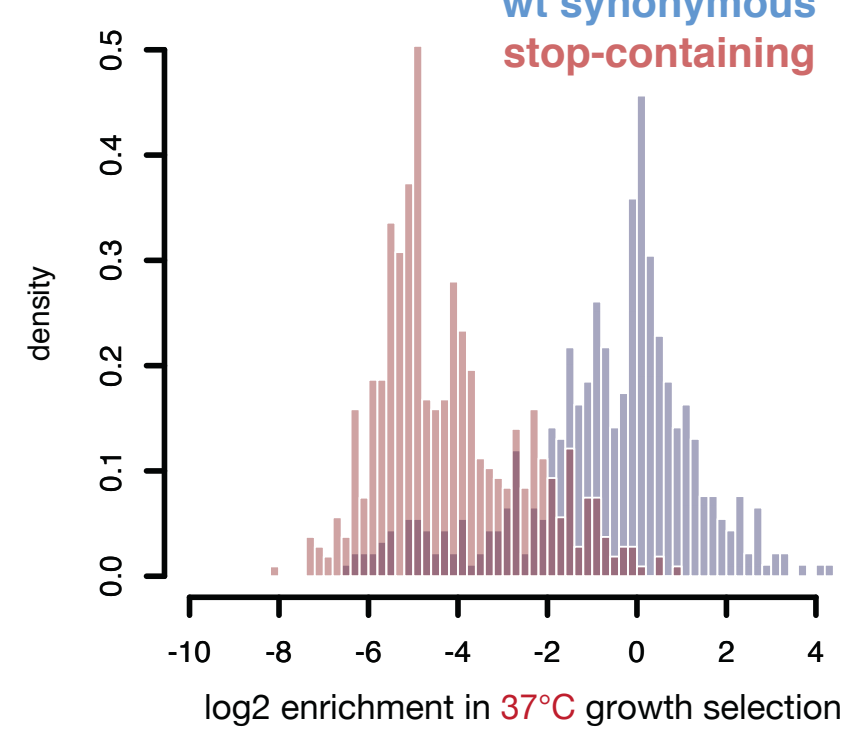

Supplementary Figure 3. Synonymous Hsf1 variants show enrichment scores centered around zero. Histograms showing the log2 fitness scores of variants with synonymous codon changes to wild-type (blue) or variants containing stop codons (red) after growth at $37^{\circ} \mathrm{C}$. 

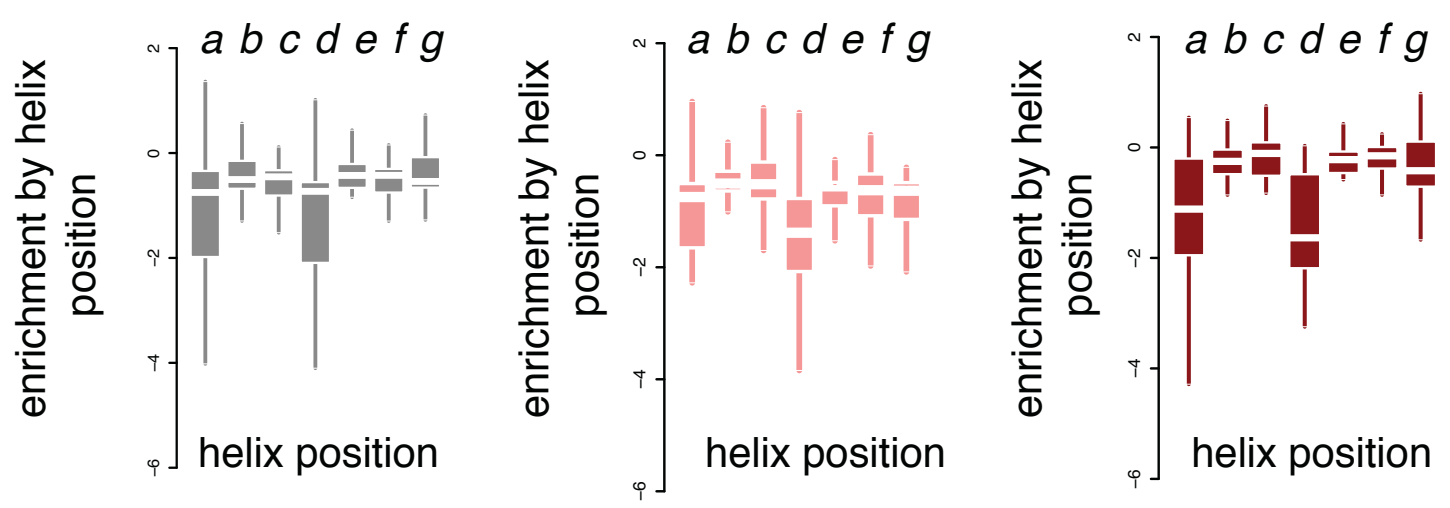

Supplementary Figure 4. Small or charged substitutions do not have cumulatively negative effects outside of helix positions $\boldsymbol{a}$ and $\boldsymbol{d}$. Boxplots showing log2 DMS enrichment scores of variants with substitutions to small or charged (K,R,D,E,S,G) residues at each site grouped by helix position (x-axis). Boxplot lines indicate median enrichment scores. 
bioRxiv preprint doi: https://doi.org 0.1101/2020.05.23.112250; this version posted May 25, 2020. The copyright holder for this preprint (which
wassinotacertified by peer rewew the author/funder, who has granted bioRxiv a license to display the preprint in perpetuity. It is made
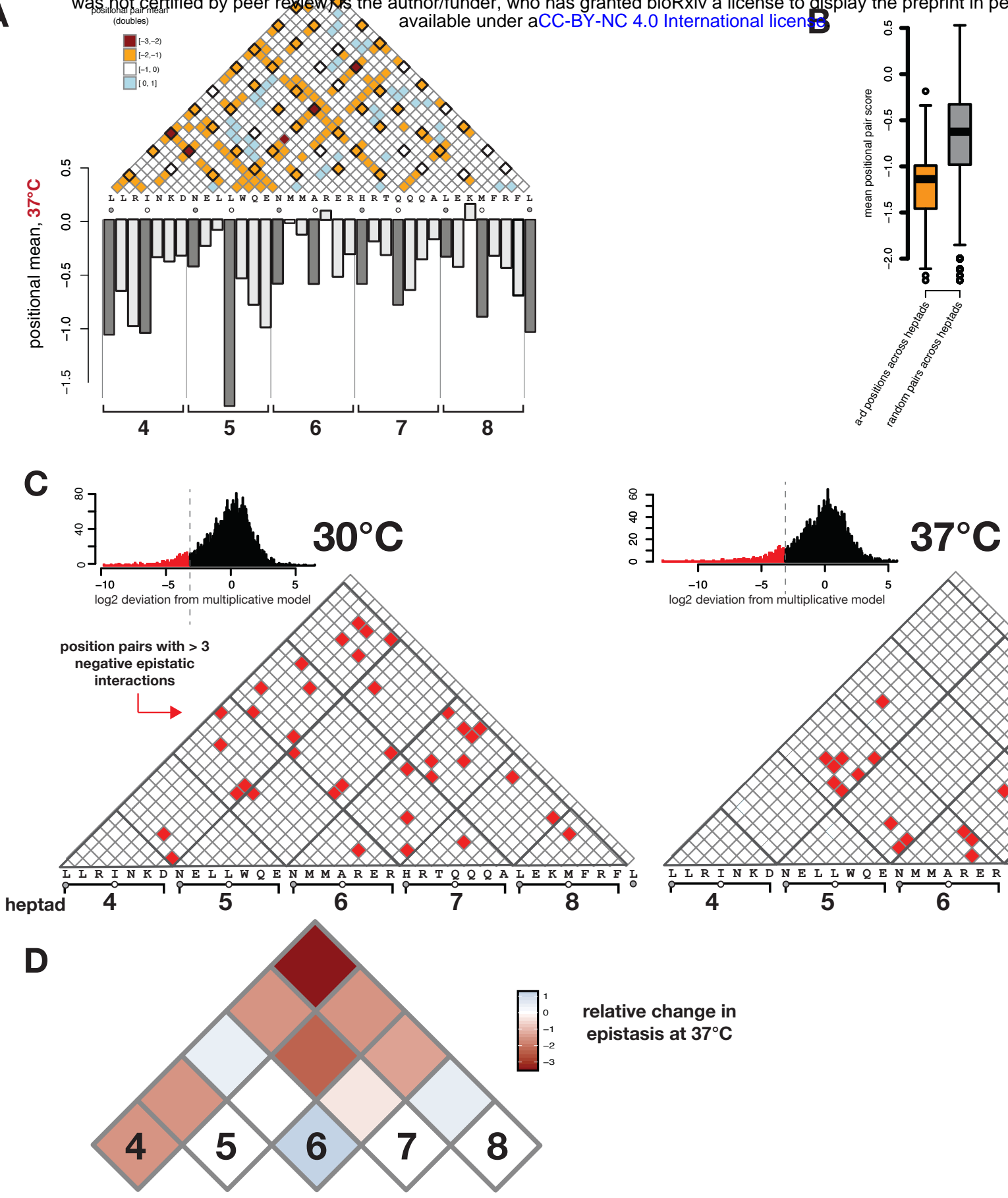

Supplementary Figure 5. Analysis of double mutants supports the functional role of a and d positions and reveals patterns of epistatically-interacting sites underlying fewer long-distance contacts at $37^{\circ} \mathrm{C}$. (A) Heatmap showing mean enrichment scores at $37^{\circ} \mathrm{C}$ of all variants found at each pair of sites. Darker shades of orange indicate site pairs for which the double mutants were more deleterious. Positional means generated for single amino acid substitutions at each residue (as in Fig. 3) are shown below for reference, with heptads indicated below. (B) Boxplot showing the mean enrichment scores of double mutants at a-d positions are more deleterious than pairs of mutations at other helix positions. (C) Pairs of sites are marked (red) where $>3$ double mutants show scores more negative than expected from multiplication of each constituent single (histogram shown above). The pattern of site pairs showing negative epistasis differs between $30^{\circ} \mathrm{C}$ (left) and $37^{\circ} \mathrm{C}$ (right). (D) The difference in epistasis scores between the two temperatures is presented as a heat map by heptad (4-8). Epistasis observed at distant heptads (e. g. between heptads 4 and 8 ) is reduced at $37^{\circ} \mathrm{C}$ compared to $30^{\circ} \mathrm{C}$. 

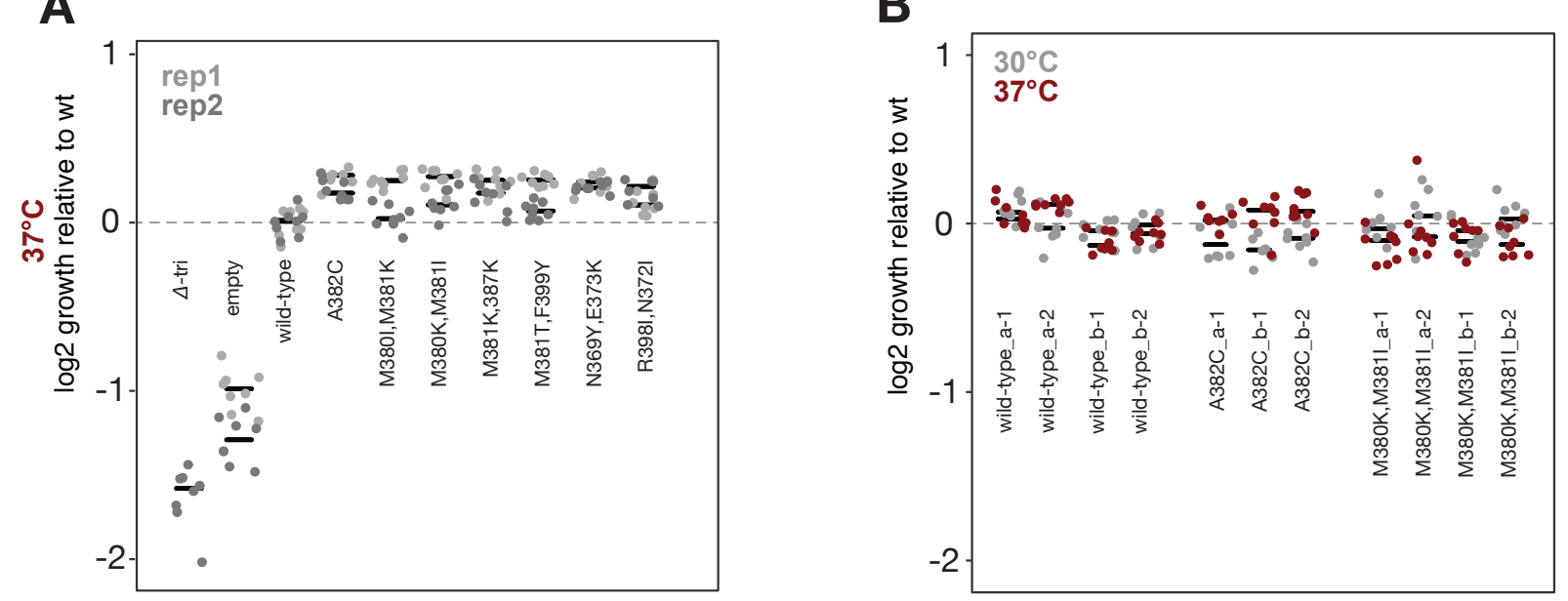

Supplementary Figure 6. Growth rate validation of variants from large screen. (A) Seven HSF1 variants identified in the DMS were independently validated in overnight plate reader growth experiments. HSF1 variants were expressed in the conditions used during the DMS: endogenous HSF1 was repressed with ATc and variant HSF1 was expressed from a plasmid with the native HSF1 promoter. HSF1 sequence contained the silent restriction enzyme sites used in DMS cloning. Dots represent technical replicate wells. Rep1 (light gray) and rep2 (dark gray) represent separate experiments performed with independent plasmid transformants. Data from rep2 is reproduced in Fig. 4C. The maximum slope of the growth curves is presented relative to the average rescue of a plasmid expressing wild-type HSF1. (B) HSF1 variants were integrated into the chromosomal HSF1 locus, under the native promoter and absent the silent restriction cloning sites. Clones from two different integration events were isolated for each genotype using URA3 marker selection ( $\mathrm{a}$ and $\mathrm{b}$ for each genotype) after which URA3 was eliminated with counterselection (see Methods) to generate clones 1 and 2 of each integrated genotype. Overnight plate reader growth experiments were performed as above, at temperatures $30^{\circ} \mathrm{C}$ and $37^{\circ} \mathrm{C}$. Individual dots represent technical replicate wells. 
bioRxiv preprint doi: https://doi.org/10.1101/2020.05.23.112250; this version posted May 25, 2020. The copyright holder for this preprint (which

A was not certified by peer review) is the author/funder, who has granted bioRxiv a license to display the preprint in perpetuity. It is made
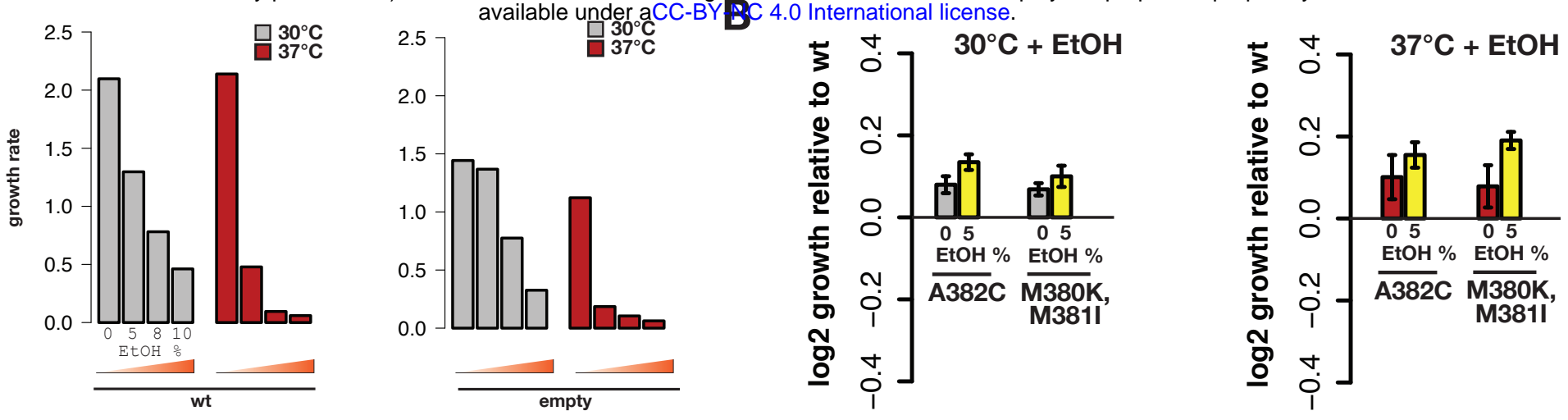

Supplementary Figure 7. HSF1 variants improve growth under temperature and ethanol stress. (A) Yeast strains with endogenous HSF1 repressed and rescued with plasmid expression of either wild-type Hsf1 (wt) or an empty vector were grown in the presence of ethanol at $0 \%, 5 \%$, $8 \%$, or $10 \%$. Overnight growth was assessed in a plate reader at $30^{\circ} \mathrm{C}$ or $37^{\circ} \mathrm{C}$ and the maximum growth velocity is presented. (B) Yeast strains were assessed as above but with expression of two different HSF1 variants: A382C and M380K,M381I, both of which were identified for their ability to confer enhanced growth at $37^{\circ} \mathrm{C}$ (Fig. $4 \mathrm{C}$ ). The log2 of their maximum growth velocity relative to wild type is presented for growth in $0 \%$ or $5 \%$ ethanol at $30^{\circ} \mathrm{C}$ or $37^{\circ} \mathrm{C}$. (Error bars represent standard error of the mean.) 


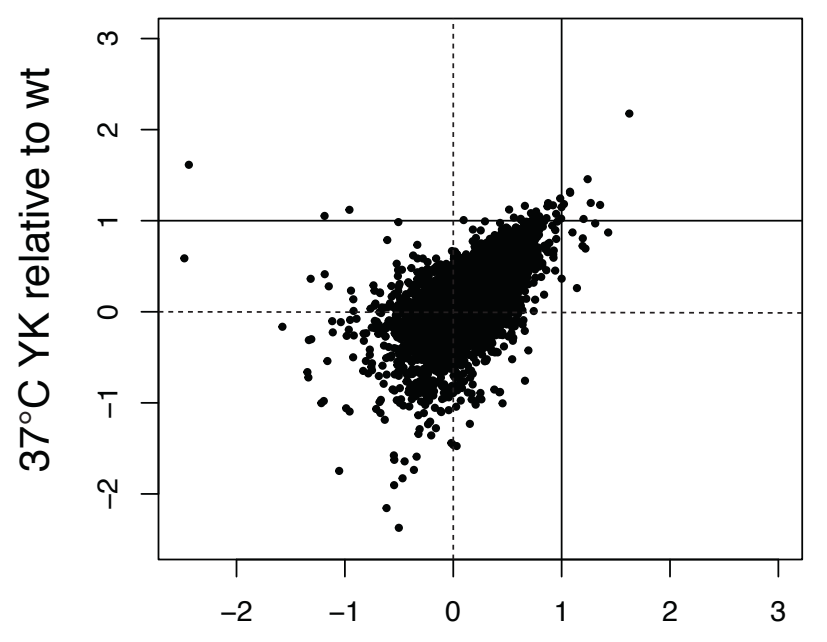

$37^{\circ} \mathrm{C} \mathrm{KI}$ relative to $\mathrm{wt}$

Supplementary Figure 8. HSF1 variants conferring enhanced growth phenotype showed similar patterns of upregulated genes under temperature stress. Scatterplot showing log2 relative expression level of each gene in M380K,M381I (KI) and N369Y,E373K (YK) variants at $37^{\circ} \mathrm{C}$. Dotted guidelines represent no change in expression relative to wild type and solid guidelines indicate 2-fold changes in expression. 


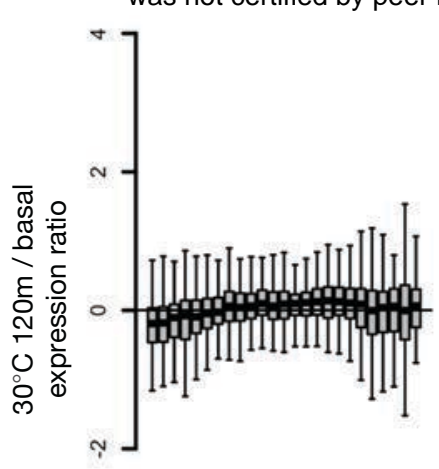

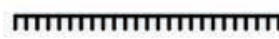

composite heat-shock annotation score

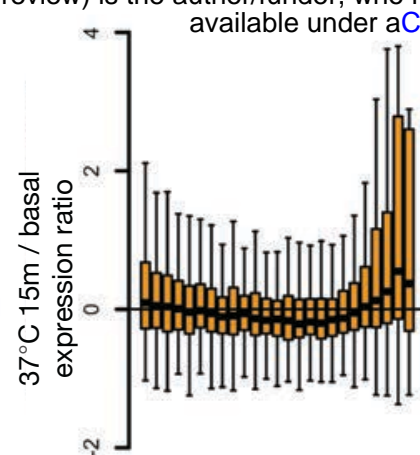

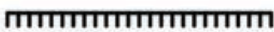

composite heat-shock annotation score

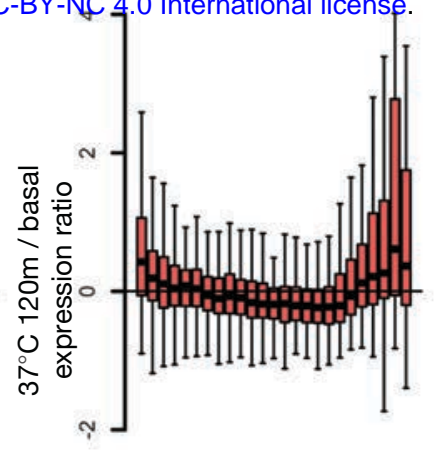

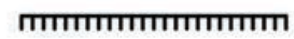

composite heat-shock annotation score

B
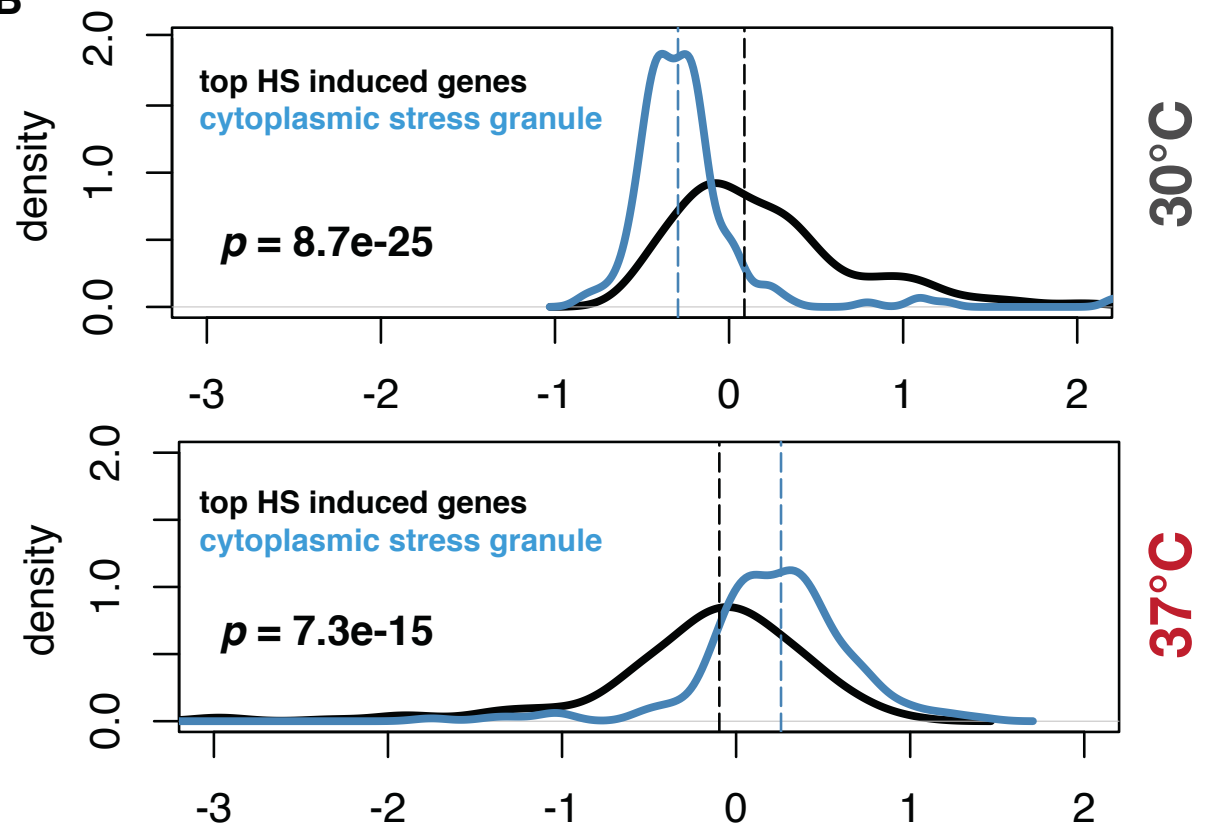

log2 expression relative to wild-type

Supplementary Figure 9. Yeast with expression of wild-type HSF1 activate a core heat-shock response similar to previous data measuring gene expression in cells shifted to $37^{\circ} \mathrm{C}$; variants show similar coreheat-shock response but alter expression of cytoplasmic stress granulerelated genes at both temperatures. (A) Boxplots showing the RNA-seq expression levels of genes grouped by their composite rank among several expression studies in the Saccharomyces Genome Database testing $37^{\circ} \mathrm{C}$ induction. Each bin contains 200 genes, and the highest rank bin (further right on $\mathrm{x}$-axis) includes the genes with the highest $37^{\circ} \mathrm{C}$ induction across all previous expression studies. For each bin, a boxplot of RNA-seq expression values are shown. The furthest left plot shows the RNAseq expression values for cells harvested from the $30^{\circ} \mathrm{C}$ (non-stress) condition 120 minutes after repression of wild-type HSF1. In this case, bins containing frequently heat-induced genes show no significant expression increase. In contrast, the middle and right plots showing RNA-seq expression values from the $37^{\circ} \mathrm{C}$ (stress) condition 15 minutes and 120 minutes after repression of wild-type Hsf1, respectively, show increased expression of genes within bins containing the genes most frequently upregulated at $37^{\circ} \mathrm{C}$ in previous studies. (B) Density histograms showing the expression levels of two key sets of genes expressed in the two HSF1 variants (M380K,M381I and N369Y,E373K) relative to wild type. One set represents heat-shock induced genes from the top-ranked bin in (A) (black line) and the second represents all genes with the cytoplasmic stress granule annotation (blue line). While expression of cytoplasmic stress granule genes differs from wild type in the exceptional variants at both $30^{\circ} \mathrm{C}$ and $37^{\circ} \mathrm{C}$, expression of the top heat-shock induced genes remains similar. 
